Jussi Salmi, Andreas Richter, and Visa Koivunen. 2009. Detection and tracking of MIMO propagation path parameters using state-space approach. IEEE Transactions on Signal Processing, volume 57, number 4, pages 1538-1550.

(C) 2009 IEEE

Reprinted with permission.

This material is posted here with permission of the IEEE. Such permission of the IEEE does not in any way imply IEEE endorsement of any of Helsinki University of Technology's products or services. Internal or personal use of this material is permitted. However, permission to reprint/republish this material for advertising or promotional purposes or for creating new collective works for resale or redistribution must be obtained from the IEEE by writing to pubs-permissions@iee.org.

By choosing to view this document, you agree to all provisions of the copyright laws protecting it. 


\title{
Detection and Tracking of MIMO Propagation Path Parameters Using State-Space Approach
}

\author{
Jussi Salmi, Student Member, IEEE, Andreas Richter, Senior Member, IEEE, and \\ Visa Koivunen, Senior Member, IEEE
}

\begin{abstract}
This paper describes a novel approach for detection, estimation and tracking of multiple-input multiple-output (MIMO) radio propagation parameters from multidimensional channel sounding measurements. A realistic state-space model is developed for the purpose, and the extended Kalman filter (EKF) is applied in a particular computationally efficient form to track the geometrical double-directional propagation path parameters. The observation model utilizes the dense multipath component (DMC), describing the distributed scattering in the channel, as part of the underlying noise process. The DMC model assumes an exponential profile in delay, and allows for an arbitrary angular distribution. In addition, a novel dynamic state dimension estimator using statistical goodness-of-fit tests is introduced. The employed methods are supported by illustrative estimation examples from MIMO channel sounding measurements.
\end{abstract}

Index Terms-Channel modeling, extended Kalman filter, MIMO channel sounding measurements, parameter estimation, state-space modeling.

\section{INTRODUCTION}

I $\mathrm{N}$ this paper, the problem of multidimensional high-resolution propagation parameter estimation and tracking from channel sounding measurements is addressed. In particular, the focus is on MIMO systems and mobile radio channels. The development of realistic radio channel models from multidimensional channel measurements is essential for supporting transceiver design and network planning for upcoming wireless MIMO communication systems. Realistic dynamic models for mobile wireless systems should also be based on dynamic measurements. The observed data are processed to estimate the parameters of geometrical double-directional channel models [1]. The development and verification of realistic statistics for parametric channel models depends heavily on such estimates.

Manuscript received December 05, 2007; revised October 21, 2008. First published December 31, 2008; current version published March 11, 2009. The associate editor coordinating the review of this manuscript and approving it for publication was Dr. Gerald Matz. The research is partially funded by WILATI project. J. Salmi acknowledges the financial support of the Finnish Technology Promotion Foundation (TES), Emil Aaltonen Foundation, Finnish Society of Electronics Engineers (EIS), HPY Research Foundation, and Nokia Foundation.

J. Salmi and V. Koivunen are with Department of Signal Processing and Acoustics, Helsinki University of Technology/SMARAD CoE, FIN-02150 Espoo, Finland (e-mail: Jussi.Salmi@tkk.fi; ).

A. Richter was with Department of Signal Processing and Acoustics, Helsinki University of Technology/SMARAD CoE, FIN-02150 Espoo, Finland. He is now with Nokia Research Center, FIN-00180 Helsinki, Finland (e-mail: andreas.richter@nokia.com).

Color versions of one or more of the figures in this paper are available online at http://ieeexplore.ieee.org.

Digital Object Identifier 10.1109/TSP.2008.2011823
The extraction of the channel model parameters from measurement data has typically been based on the maximum-likelihood (ML) criterion and advanced numerical solutions. Example methods include the SAGE-based algorithms [2] (space alternating expectation maximization) or RIMAX [3] (iterative ML). ML approaches assume that data are i.i.d. However, it can be observed from measurements that the specular component of the radio channel contains typically propagation paths which persist over a relatively large number of snapshots. Furthermore, the parameters of these paths vary slowly in time. This observation suggests that the path parameters could be tracked over time in order to capture the dynamic behavior of the radio channel. Moreover, application of sequential estimation methods reduces computational complexity.

An alternative approach for capturing the dynamics of the MIMO radio channel was taken in [4]. In that contribution, the estimates from a SAGE-based [2] estimator were clustered, and the cluster positions were tracked over time. However, there are several advantages in direct sequential estimation (tracking) of propagation parameters, as follows.

- The parameters of individual paths are automatically paired, i.e., a trace of parameter estimates describing one path is obtained.

- Additional information about how parameters evolve over time including the rate of change can be obtained.

- Due to filtering the estimation error is reduced.

- Propagation path parameters can be tracked across deep fades.

- Sequential estimation reduces the computational complexity since the previous estimate can be updated using the new information captured by the current measurement. Altogether, the estimation and tracking approach provides better insight into properties of mobile MIMO radio channels with a reduced computational burden.

Commonly, the radio wave propagation is modelled using a superposition of specular-like highly concentrated paths. In this work also so-called dense multipath (DMC) [3] is taken into account. The DMC explains the parts of the channel, which cannot be estimated individually as paths, due to limited measurement apperture and the complexity of the underlying physical process. It captures a significant part of the rich scattering that is crucial for achieving all the MIMO system gains. Moreover, the model complexity is reduced. Instead of attempting to estimate a very large number of individually weak scattering components with a high-dimensional deterministic model (a superposition of propagation paths), the diffuse scattering is modeled stochastically with a reduced number 
of parameters. This type of stochastic model-along with the superposition of propagation paths-captures the essential properties of the radio channel.

In this paper, a state-space approach is used for tracking the dynamic radio propagation path parameters over time. A statespace model is derived and an extended Kalman filter (EKF) is applied for the parameter estimation of the nonlinear data model. Another approach for the tracking of the dynamic space, time, and frequency dependent MIMO radio channel was recently proposed in [5] based on Particle Filter concept. However, in [5] the number of tracked paths is very limited and the number of particles (5) per path is unrealistically low, leading to an increased need for particle resampling. As the dimensionality of the problem grows, these issues can be expected to increase the computational complexity accordingly. In addition, the diffuse scattering (DMC) was not considered in [5].

In this paper, the EKF is formulated in a specific, computationally attractive form, enabling scalability for large state and measurement dimensions. Realistic models for state noise covariance and propagation are developed in order to capture the underlying physical phenomena, to keep the computational complexity reasonable, and to obtain an estimator with good statistical performance in terms of estimation error (studied in [6] and [7]). In particular a new model for the polarimetric path weights is introduced, enabling the joint estimation and tracking of structural (time-delays and angles) as well as weight (polarimetric path coefficients) parameters. This paper also introduces a novel method for the dynamic adjustment of the state dimension by applying statistical hypothesis tests for the goodness-of-fit. In addition, the observation noise model describing DMC is extended to cover spatial as well as temporal properties of the scattering component in radio channels. The design of the observation and state-space model is supported by examples obtained from mobile MIMO channel sounding measurements.

The paper is structured as follows. In Section II, the concept of measurement based realistic channel modeling and parameter estimation in MIMO systems is discussed. Section III addresses the philosophy behind the parametrization for the dynamic state-space model. In Section IV, the state-space model is derived. Section $\mathrm{V}$ describes the sequential estimation procedure stemming from the EKF. Model order estimation and the adjustment of state dimension are considered as well. Section VI presents estimation results with real-world data, and Section VII concludes the paper.

The notation used throughout the paper is as follows.

- Upper case letters denote constants, and lower case denote scalars.

- Boldface upper case letters (Roman or Greek) denote matrices.

- Boldface lower case letters denote vectors (column, if not stated otherwise).

- Superscripts ${ }^{\mathrm{T}}$ and ${ }^{\mathrm{H}}$ denote matrix transpose and Hermitian transpose, respectively.

- Subscripts ${ }_{\mathrm{T}}, \mathrm{R}$, and ${ }_{f}$ refer to transmitter, receiver and frequency domains, respectively.

- The operators $\otimes, \odot$, and $\diamond$ denote Kronecker, Schur, and Khatri-Rao products, respectively.

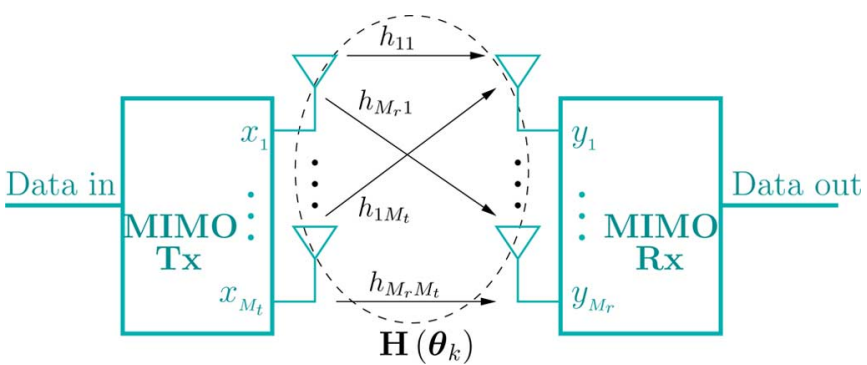

Fig. 1. Description of the multiantenna communication, i.e., MIMO principle. Our focus is on the estimation of the parameters $\boldsymbol{\theta}_{k}$ describing the time varying channel matrix $\mathbf{H}$.

- Vector $\hat{\boldsymbol{\theta}}_{k}$ denotes an estimate of $\boldsymbol{\theta}$ at discrete time instant $k$.

- $\mathbf{J}\left(\hat{\boldsymbol{\theta}}_{k}, \mathbf{R}_{k}\right)$ denotes a matrix valued function $\mathbf{J}$ of vector $\hat{\boldsymbol{\theta}}_{k}$ and matrix $\mathbf{R}_{k}$.

- Matrix $\mathbf{I}_{P}$ denotes a $P \times P$ identity matrix, $\mathbf{1}_{P}$ denotes a $P \times 1$ vector of ones, and $\mathbf{0}$ denotes a matrix of zeros with appropriate dimensions.

\section{MIMO CHANNEL MODELING}

\section{A. Fading Radio Channels}

It is well known that in mobile communications multipath propagation severely affects the quality of the received signal in any radio link. The base station receives the transmitted signal from the mobile station via a line-of-sight (LOS) path, if present, and multiple delayed copies from reflected, scattered, or diffracted paths. In general, these paths impinge at the base station antenna from different directions of arrival (DOA) corresponding to specific directions of departure (DOD) at the mobile station.

The superposition of the received signals causes frequencyand space-selective fading. Due to user mobility and possible movement of scatterers in the propagation environment, the fading is also time-varying. Slow fading arises from path shadowing (the LOS may be temporarily obscured by buildings etc.), whereas fast fading is caused by self-interference under multipath propagation. In general, the propagation path parameters of interest, including time delays of arrival $\tau_{p}$ (TDoA), the directions of arrival (DoA) $\varphi_{R, p}$ (azimuth), $\vartheta_{R, p}$ (elevation), the directions of departure (DoD) $\varphi_{T, p}$ (azimuth), $\vartheta_{T, p}$ (elevation), as well as the total number of relevant paths $P$, are changing over time. This results in a random time-variant channel impulse response (CIR).

Traditionally, fading is considered as a serious degradation of the radio link. Recently, it has been discovered that using multiple antennas at both transmitter and receiver combined with appropriate space-time signal processing, the system performance of mobile radio systems can be significantly enhanced. Multiple-Input Multiple-Output (MIMO) communication systems [8], as depicted in Fig. 1, can benefit from dispersive fading channels. The expected benefits include increased capacity, range, and radio link quality as a result of diversity, array and multiplexing gains, as well as interference reduction using advanced signal processing. The goal of MIMO 


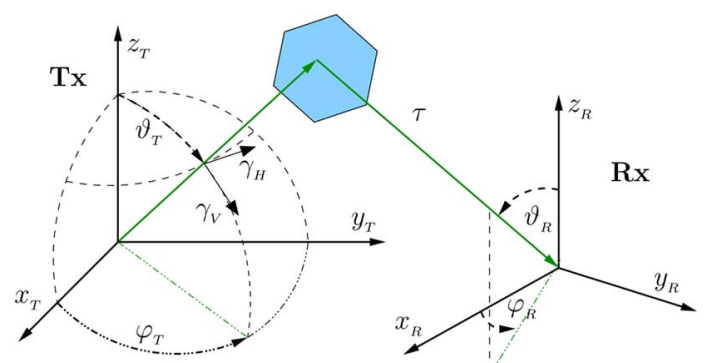

Fig. 2. Double directional radio channel model parameters for a single propagation path. Tx denotes transmitter and Rx receiver.

space-time signal processing is to optimally exploit the spatial diversity of multipath channels to obtain the above gains.

\section{B. Double Directional MIMO Channel Model}

To extract the spatial and temporal information of the MIMO radio channel from measurements, the double directional channel model [1] is employed. A part of the channel response can be expressed as a superposition of $P$ propagation paths

$$
\begin{array}{r}
\mathbf{H}(f)=\sum_{p=1}^{P}\left\{\mathbf{B}_{\mathrm{R}}\left(\varphi_{R, p}, \vartheta_{R, p}\right) \cdot\left[\begin{array}{ll}
\gamma_{H H, p} & \gamma_{H V, p} \\
\gamma_{V H, p} & \gamma_{V V, p}
\end{array}\right]\right. \\
\left.\cdot \mathbf{B}_{\mathrm{T}}\left(\varphi_{T, p}, \vartheta_{T, p}\right)^{\mathrm{T}} \cdot \mathrm{e}^{-\mathrm{j} 2 \pi f \tau_{p}}\right\}
\end{array}
$$

where $\mathbf{B}_{\mathrm{R}}$ and $\mathbf{B}_{\mathrm{T}}$ are the nonlinear mappings of the angles of arrival $\left(\varphi_{\mathrm{R}}, \vartheta_{\mathrm{R}}\right)$ and departure $\left(\varphi_{\mathrm{T}}, \vartheta_{\mathrm{T}}\right)$ to the antenna array responses. The parameters $\gamma_{i}$ denote the radio wave polarization coefficients (horizontal-to-horizontal $(\mathrm{HH})$, horizontal-tovertical ( $\mathrm{HV}$ ), etc.). The meaning of the propagation path parameters is illustrated in Fig. 2.

In Section IV-C, the model for the DMC, describing the part of the channel which is not approximated using concentrated paths, is described.

\section{MIMO Channel Sounding}

MIMO radio channel measurements are obtained using channel sounders [9]. A channel sounder measures the channel at a certain bandwidth for each pair of $M_{\mathrm{T}}$ transmit (Tx) and $M_{\mathrm{R}}$ receive $(\mathrm{Rx})$ antennas. In practical channel sounding systems, due to the large number of channels $\left(M_{\mathrm{T}} \cdot M_{\mathrm{R}}=10^{2} \cdots 10^{4}\right)$, the measurements are usually performed sequentially for each $\mathrm{Tx}-\mathrm{Rx}$ antenna pair. The timing of sequential channel sounding measurements is shown in Fig. 3, where $T_{s}=1 / f_{s}$ denotes the sampling interval at sampling frequency $f_{s}$, and $T_{m}$ is the time taken to measure a full MIMO channel (snapshot). Each channel is commonly measured twice to ensure stability after switching. It should also be noted that the time between snapshots $\Delta t$ is typically large compared to $T_{m}$.

The data analyzed in this work has been measured with MEDAV's RUSK sounder [10] by Ilmenau Technical University, Ilmenau, Germany. This sounder relies on the multi carrier spread spectrum waveforms. The number of measured channels in the used setup was $M_{\mathrm{T}} \cdot M_{\mathrm{R}}=16 \cdot 16$ and the number of effective frequency samples was $M_{f}=193$ (on a bandwidth of $f_{s}=120 \mathrm{MHz}$ ). This results in $M=M_{f} M_{\mathrm{T}} M_{\mathrm{R}} \approx 5 \cdot 10^{4}$ complex samples per snapshot. The time between snapshots in

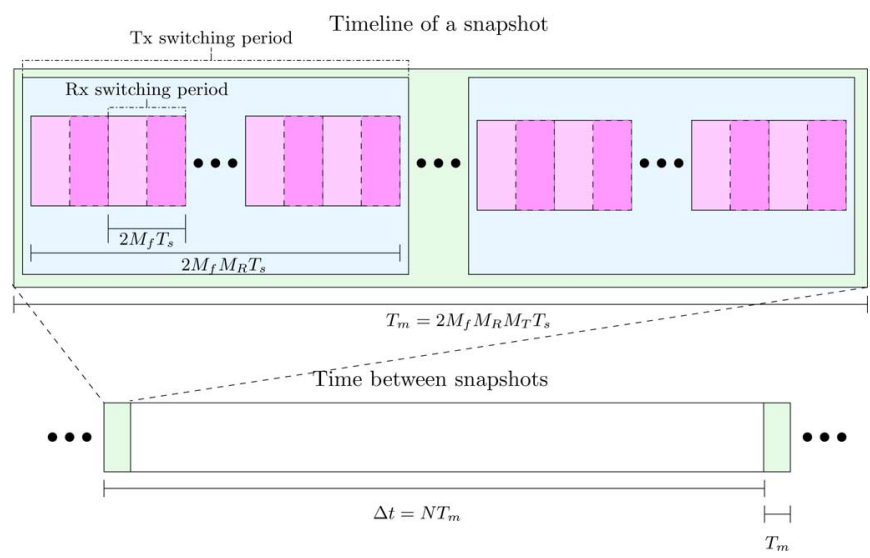

Fig. 3. Typical structure of sequentially switched MIMO channel sounding measurements.

this setup was $\Delta t=N T_{m} \approx 20 \mathrm{~ms}$, with $N=25$. The carrier frequency was $f_{c}=5.2 \mathrm{GHz}$.

\section{Parametrization of the Dynamic MiMo PROPAGATION CHANNEL}

In this section, fundamental statistics of propagation path parameters observed in MIMO channel sounding measurements and the state-space model used in this work are discussed. The state-space model presented in this paper is a tradeoff between a desired ideal description for approximating the electromagnetic propagation as a sum of discrete far-field propagation paths, versus a realistic modeling approach to achieve reliable and identifiable parameter estimates under the influence of measurement system limitations [3], [11].

The parameters describing the propagation paths are comprised of the structural parameters

$$
\boldsymbol{\mu}=\left[\begin{array}{lllll}
\boldsymbol{\tau}^{\mathrm{T}} & \boldsymbol{\varphi}_{\mathrm{T}}^{\mathrm{T}} & \boldsymbol{\vartheta}_{\mathrm{T}}^{\mathrm{T}} & \boldsymbol{\varphi}_{\mathrm{R}}^{\mathrm{T}} & \boldsymbol{\vartheta}_{\mathrm{R}}^{\mathrm{T}}
\end{array}\right]^{\mathrm{T}} \in \mathbb{R}^{5 \cdot P \times 1}
$$

related to the propagation environment geometry, and the path weight parameters

$$
\boldsymbol{\gamma}=\left[\begin{array}{llll}
\gamma_{\mathrm{HH}}^{\mathrm{T}} & \boldsymbol{\gamma}_{\mathrm{HV}}^{\mathrm{T}} & \boldsymbol{\gamma}_{\mathrm{VH}}^{\mathrm{T}} & \gamma_{V V}^{\mathrm{T}}
\end{array}\right]^{\mathrm{T}} \in \mathbb{C}^{N_{\mathrm{pol}} \cdot P \times 1} .
$$

For the full model, $N_{\mathrm{pol}}=4$ (dual polarized link ends). However, depending on the measurement setup, the number of observable path weights may be only $N_{\text {pol }}=1,2$.

\section{A. Path Weight Parametrization}

One of the challenges in parametric state-space modelling of radio channel observations is the design of a proper model for the complex path weight $\gamma$ in (3). For the state-space model, the path weights are parameterized as

$$
\alpha_{i}+\mathrm{j} \phi_{i}=\log _{\mathrm{e}}\left(\gamma_{i}\right) .
$$

This logarithmic parametrization is justified by the log-normal fading (shadowing) of the path weight magnitudes and it also facilitates tracking of the phases of the path weights. Parametrization using real and imaginary parts of the path weights would allow the parameters to be linear in the measurement equation, whereas their prediction (state transition) would be nonlinear. 


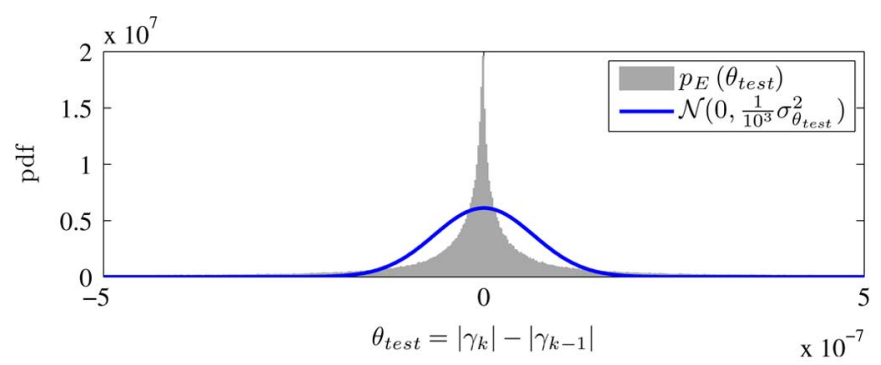

(a)

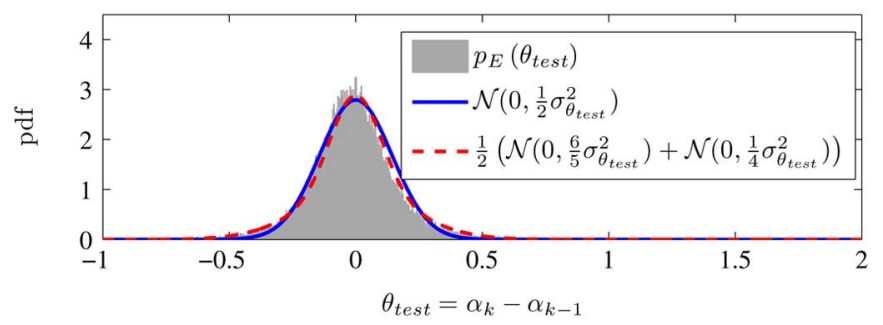

(b)

Fig. 4. Illustration how $\alpha_{i}=\log _{e}\left(\left|\gamma_{i}\right|\right)$ parametrization for the path weight yields a nearly normal distributed state transition process. Empirical densities $\left(p_{E}\right)$ are evaluated from estimated path weights from a dynamic MIMO measurement in an urban scenario [14]. (a) Density of the change in the path weight magnitude. (b) Density of the change in the logarithm of the path weight magnitude.

This conclusion stems from the fact that, for typical measurement rates and dynamics in the environment, the path weight phase can evolve to any value (from $-\pi$ to $\pi$ ) between the measurements. Hence, the real and imaginary parts have effectively zero mean over time.

1) Parametrization of Magnitude: Ideally, the model (1) decomposes the radio channel into individual multipath components, which implicitly do not suffer from fast fading resulting from superposition of paths (rays). In practice, the resolution of the measurement system limits this assumption.

Let us consider that the path weight magnitude would be parameterized using the absolute value $a=|\gamma|$ instead of its logarithm $\alpha=\log _{\mathrm{e}}(|\gamma|)$ in (4). This is illustrated in Fig. 4, where the empirical densities of the time evolution $\Delta a_{k-1}=\left|\gamma_{k}\right|-\left|\gamma_{k-1}\right|$ and $\Delta \alpha_{k-1}=\log _{\mathrm{e}}\left(\left|\gamma_{k}\right|\right)-\log _{\mathrm{e}}\left(\left|\gamma_{k-1}\right|\right)$ are shown. The transition $\Delta \alpha_{k-1}$ Fig. 4(b) has nearly normal distribution, whereas $\Delta a_{k-1}$ Fig. 4(a) has heavier tails and resembles more a Laplacian distribution. Using the logarithm of the magnitude $(\alpha=$ $\left.\log _{e}(|\gamma|)\right)$ as a parametrization yields an additive random walk prediction model instead of a multiplicative one (as would be the case for $a=|\gamma|$ ). Hence, the magnitude fluctuation is better captured. This observation agrees well with the approach used e.g., in the COST 273 MIMO-radio channel model [12]. One can also observe from Fig. 4(b), that a contaminated normal distribution [13] would have an even better fit, which results from the fact that the estimates originate from nonstationary measurements, where the variance of the process is not constant.

2) Parametrization of Phase: The phase change of a (narrowband) propagation path component during one measurement cycle (duration $\Delta t$ ) is related to the carrier frequency $f_{c}$, relative motion $v_{r}$ (projection of the terminal or scatterer movement, or both), and the path length (delay) change as

$$
\left|\phi_{k}-\phi_{k-1}\right| \approx 2 \pi f_{c}\left|\Delta \tau_{k}\right|=2 \pi f_{c} \Delta t\left|v_{r, k}\right| / c
$$

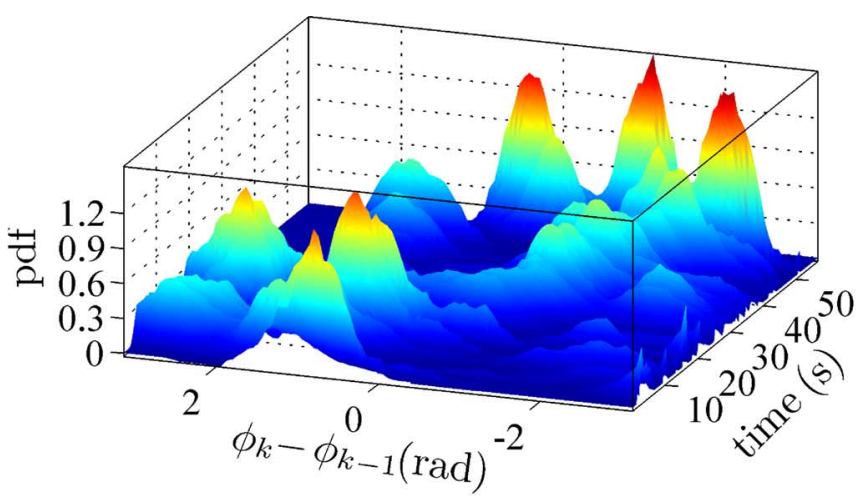

(a)

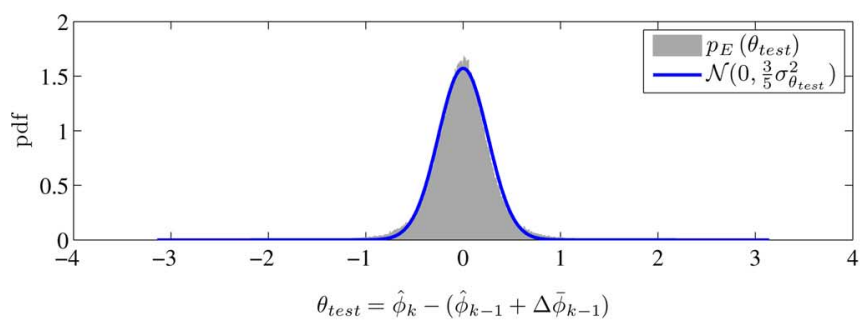

(b)

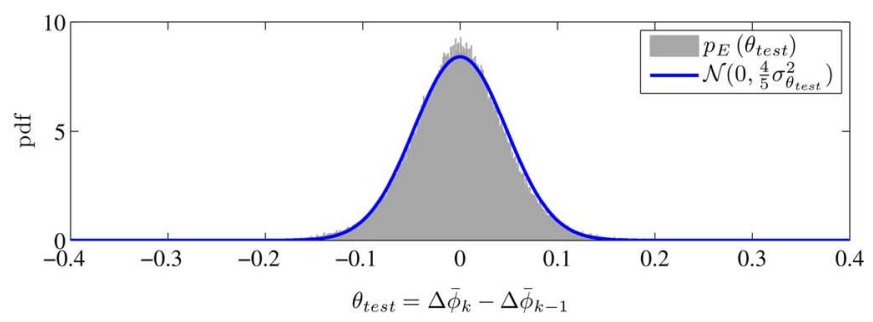

(c)

Fig. 5. Empirical density of path weight change without and with prediction using estimated $\Delta \bar{\phi}$ parameters. Empirical densities $\left(p_{E}\right)$ are evaluated from estimated path weights from a dynamic MIMO measurement in an urban scenario [14]. (a) Density of the change in the path weight phase over a measurement. (b) Density of the change of the fast varying component of $\Delta \phi$ after prediction. (c) Density of the change of the slow varying component of $\Delta \phi$, i.e., of the short term average $\Delta \bar{\phi}$, after prediction.

where $c$ denotes the propagation speed of light. For example a system with $f_{c}=5 \mathrm{GHz}, v_{r}=1 \mathrm{~m} / \mathrm{s}$ and $\Delta t=20 \mathrm{~ms}$ yields $\left|\phi_{k}-\phi_{k-1}\right|=(2 / 3) \pi=120^{\circ}$. The relative frequency of $\Delta \phi_{k}=\phi_{k}-\phi_{k-1}$ observed in a MIMO channel sounding measurement is shown in Fig. 5(a). One would have to specify the state noise variance to account for this variation caused by a large deterministic change in phase from $k-1$ to $k$. In practice the variation is so large that e.g., an EKF would fail to track the phase reliably, due to the linearization used in the filter. The solution is to include the slowly varying component $\Delta \bar{\phi}_{k}$ (short term average) of the phase change $\Delta \phi_{k}$, in the phase-model.

In Fig. 5, the models for the path-weight phase, with and without $\Delta \bar{\phi}_{k}$, are compared. In practice the phase change $\Delta \phi_{k}$ is (in average over time) almost uniformly distributed in the interval $\langle-\pi, \pi\rangle$ as shown in Fig. 5(a). However, $\phi_{k}-\left(\phi_{k-1}+\right.$ $\left.\Delta \bar{\phi}_{k-1}\right)$ can be well approximated with a zero-mean Normal distribution as shown in Fig. 5(b). The statistics of the change of $\Delta \bar{\phi}_{k}$ is shown in Fig. 5(c). As a conclusion, including $\Delta \bar{\phi}_{k}$ in the state enables reliable prediction of the path weights by allowing the linearization of the model using the first order Taylorseries approximation, employed in the EKF. 


\section{B. Doppler Shift in Channel Sounding}

The phase evolution of the signal $\Delta \bar{\phi}$ could, in principle, be interpreted as a normalized Doppler shift $\mu_{\alpha_{D}}$. The relation of $\Delta \bar{\phi}$ and the Doppler shift $\alpha_{D}$ is given by $\Delta \bar{\phi}=\arg \left(2 \pi \alpha_{D} \Delta t\right)$, where $\arg (\bullet)$ denotes the principal angle. However, due to the possible nonlinearity of the observed phase evolution, the estimation of $\hat{\mu}_{\alpha_{D}}$ is ambiguous in $\hat{\mu}_{\alpha_{D}}=\mu_{\alpha_{D}}+n \cdot 2 \pi, n \in \mathcal{Z}$. This ambiguity is irrelevant while tracking $\Delta \bar{\phi}$, and the Doppler shift can be inferred in postprocessing with careful analysis since the trajectories and velocities of the receiver and transmitter are known.

Due to the sequential measurement of the channels (see Fig. 3), the effect of the Doppler shift could also be included in a straightforward manner (see [3]) in the measurement model of a single MIMO snapshot (Section IV-B). However, in the measurement setup considered in this paper (see Section II-C) this effect is negligible. The time taken to measure a snapshot is $T_{m}=2 M_{f} M_{\mathrm{T}} M_{\mathrm{R}} T_{s} \approx 0.82 \mathrm{~ms}$. For a relative velocity of $v_{r}=1 \mathrm{~m} / \mathrm{s}$, and carrier frequency $f_{c}=5.2 \cdot 10^{9}$, the resulting phase difference between the first and the last channel is $\Delta \phi_{m}=2 \pi v_{r} / c_{0} f_{c} T_{m} \approx 0.01 \mathrm{rad}$. This is effectively hidden in the phase noise of the sounding system (see [15] and [16]). Hence, with these assumptions, the Doppler shift can not be estimated reliably from a single snapshot. The Doppler correction becomes a necessity in scenarios with high mobility relative to the snapshot measurement time. In that case, even the sequence of measuring the channels could be optimized; see [17].

\section{Modeling the Dynamics of the System}

To describe the state-space model of the propagation paths, we propose the following structure for realistic and identifiable modeling of the dynamics. The state vector (parameters to track) at time $k$ is given by

$$
\boldsymbol{\theta}_{k}=[\underbrace{\boldsymbol{\mu}^{\mathrm{T}}}_{5 P \times 1} \underbrace{\Delta \overline{\boldsymbol{\mu}}^{\mathrm{T}}}_{5 P \times 1} \underbrace{\boldsymbol{\alpha}^{\mathrm{T}}}_{4 P \times 1} \underbrace{\boldsymbol{\phi}^{\mathrm{T}}}_{4 P \times 1} \underbrace{\Delta \overline{\boldsymbol{\phi}}^{\mathrm{T}}}_{P \times 1}]^{\mathrm{T}}
$$

where also the rate of change of the structural parameters $\Delta \overline{\boldsymbol{\mu}} \in$ $\mathbb{R}^{5 \cdot P \times 1}$ is introduced. The evolution of the state from a measurement to the next one is modeled as

$$
\begin{aligned}
\boldsymbol{\mu}_{k} & =\boldsymbol{\mu}_{k-1}+\Delta \overline{\boldsymbol{\mu}}_{k-1}+\mathbf{v}_{\mu_{k}} \\
\Delta \overline{\boldsymbol{\mu}}_{k} & =\Delta \overline{\boldsymbol{\mu}}_{k-1}+\mathbf{v}_{\Delta \bar{\mu}_{k}} \\
\boldsymbol{\alpha}_{i k} & =\boldsymbol{\alpha}_{i k-1}+\mathbf{v}_{\alpha k} \\
\boldsymbol{\phi}_{i k} & =\boldsymbol{\phi}_{i k-1}+\Delta \overline{\boldsymbol{\phi}}_{k-1}+\mathbf{v}_{\phi_{k}} \\
\Delta \overline{\boldsymbol{\phi}}_{k} & =\Delta \overline{\boldsymbol{\phi}}_{k-1}+\mathbf{v}_{\Delta \overline{\boldsymbol{\phi}}_{k}}
\end{aligned}
$$

where the vectors $\mathbf{v}_{k}$ denote the state noise. The last parameter $\Delta \bar{\phi}$ is the short-term average rate of change of the path weight phases $\phi_{i}$, which is common for all polarization coefficients of a path. Tracking the rate of change parameters $(\Delta \bar{\mu}$ and $\Delta \bar{\phi})$ is related to ARIMA time series modeling [18] (with autoregressive, moving average, and differencing orders of 1,0 , and 1 , respectively). This observation results from the fact that the $\Delta$-parameters are essentially used for differentiating the respective parameters over time, yielding a stationary process for the state evolution.
The continuous-time dynamic equation [19] for a single path can be expressed as

$$
\begin{aligned}
\frac{\mathrm{d} \boldsymbol{\theta}(t)}{\mathrm{d} t}=\underbrace{\left[\begin{array}{ccccc}
\mathbf{0} & \mathbf{I}_{5} & \mathbf{0} & \mathbf{0} & \mathbf{0} \\
\mathbf{0} & \mathbf{0} & \mathbf{0} & \mathbf{0} & \mathbf{0} \\
\mathbf{0} & \mathbf{0} & \mathbf{0} & \mathbf{0} & \mathbf{0} \\
\mathbf{0} & \mathbf{0} & \mathbf{0} & \mathbf{0} & \mathbf{1}_{4} \\
\mathbf{0} & \mathbf{0} & \mathbf{0} & \mathbf{0} & 0
\end{array}\right]}_{\mathbf{F}} \underbrace{\left[\begin{array}{c}
\boldsymbol{\mu}(t) \\
\Delta \overline{\boldsymbol{\mu}}(t) \\
\boldsymbol{\alpha}(t) \\
\boldsymbol{\phi}(t) \\
\Delta \bar{\phi}(t)
\end{array}\right]}_{\boldsymbol{\theta}} \\
+\underbrace{\left[\begin{array}{cccc}
\mathbf{0} & \mathbf{0} & \mathbf{0} & \mathbf{0} \\
\mathbf{I}_{5} & \mathbf{0} & \mathbf{0} & \mathbf{0} \\
\mathbf{0} & \mathbf{I}_{4} & \mathbf{0} & \mathbf{0} \\
\mathbf{0} & \mathbf{0} & \mathbf{I}_{4} & \mathbf{0} \\
\mathbf{0} & \mathbf{0} & \mathbf{0} & 1
\end{array}\right]}_{\mathbf{L}} \mathbf{w}(t)
\end{aligned}
$$

where the rate of change of the structural parameters $(\Delta \overline{\boldsymbol{\mu}})$ as well as the path weight parameters $(\boldsymbol{\alpha}, \boldsymbol{\phi}$ and $\Delta \bar{\phi})$ are perturbed by a white Normal distributed process $\mathbf{w}(t)$. The process $\mathbf{w}(t)$ has a power spectral density

$$
\mathbf{W}=\left[\begin{array}{cccc}
\operatorname{diag}\left(\mathbf{q}_{\Delta \bar{\mu}}\right) & \mathbf{0} & \mathbf{0} & \mathbf{0} \\
\mathbf{0} & q_{\alpha} \mathbf{I}_{4} & \mathbf{0} & \mathbf{0} \\
\mathbf{0} & \mathbf{0} & q_{\phi} \mathbf{I}_{4} & \mathbf{0} \\
\mathbf{0} & \mathbf{0} & \mathbf{0} & q_{\Delta \bar{\phi}}
\end{array}\right]
$$

where $\operatorname{diag}(\bullet)$ denotes a diagonal matrix with its vector argument as the diagonal elements. This choice for the process noise model is motivated by the assumption that the noise dynamics of the structural parameters (2) are governed by the (macro-scale) motion of terminals and/or scatterers in the system (no $q_{\mu}$ in (9)), whereas the path weight parameters are in addition subjected to surface roughness (e.g., in reflections from walls), antenna vibration, and other (micro-scale) disturbances (modeled by $q_{\alpha}$ and $q_{\phi}$ ).

The values of the power spectral density $q$ in (9) are essentially filter design parameters. The selection and fine tuning of these should be done according to the expected kinematics in the system (for $\mathbf{q}_{\Delta \bar{\mu}}$ and $q_{\Delta} \overline{\boldsymbol{\phi}}$ ), empirically evaluated fading statistics (for $q_{\alpha}, q_{\phi}$ ), as well as balancing between smoothness of the filtered estimates versus faster tracking. The former leads to losing track of paths, whereas the latter gives higher variance but enables robust performance and longer tracks. In addition, the state noise term captures all the modeling uncertainties. Such uncertainties are bound to be present because of highly complicated propagation environments. For further reading on the subject, see [19].

\section{State-Space Model}

State-space modeling of radio channel propagation parameters is based on the observation that the parameters, if properly chosen, evolve slowly w.r.t. the measurement interval, i.e., they are correlated in time. The process can be described using a Gauss-Markov model [20], i.e., denoting the state $\boldsymbol{\theta}$ of the system at time $k$ as $\boldsymbol{\theta}_{k}$, the probability (density) of the next state $\boldsymbol{\theta}_{k+1}$ depends only on the current state $p\left(\boldsymbol{\theta}_{k+1} \mid \boldsymbol{\theta}_{k}, \boldsymbol{\theta}_{k-1} \ldots \boldsymbol{\theta}_{0}\right)=p\left(\boldsymbol{\theta}_{k+1} \mid \boldsymbol{\theta}_{k}\right)$, and is normal distributed. The state-space model used in this paper consist of the linear state equation, describing the dynamic behavior 
of the propagation parameters of $P$ paths, and a nonlinear measurement equation, mapping the double-directional model parameters to the channel sounder output data.

\section{A. State Equations}

The state vector is given by (6)

$$
\boldsymbol{\theta}_{k}=[\underbrace{\boldsymbol{\mu}^{\mathrm{T}}}_{5 P \times 1} \underbrace{\Delta \overline{\boldsymbol{\mu}}^{\mathrm{T}}}_{5 P \times 1} \underbrace{\boldsymbol{\alpha}^{\mathrm{T}}}_{4 P \times 1} \underbrace{\boldsymbol{\phi}^{\mathrm{T}}}_{4 P \times 1} \underbrace{\Delta \overline{\boldsymbol{\phi}}^{\mathrm{T}}}_{\boldsymbol{P} \times 1}]^{\mathrm{T}}
$$

i.e., the number of parameters per path in the state is $L=19$. In channel sounding the observation interval $\Delta t$ is usually constant. Thus, the time between observations can be normalized to one and the discrete-time (linear and time invariant) state transition and state-noise covariance matrices can be solved in closed form from (8) and (9) using the matrix fraction decomposition (see the Appendix) [21]-[23].

The state transition matrix (for a single path, $P=1$ ) is given by (8) and (37) as

$$
\boldsymbol{\Phi}_{\left.\right|_{P=1}}=\left[\begin{array}{ccccc}
\boldsymbol{\Phi}_{\mu} & \mathbf{I}_{5} & \mathbf{0} & \mathbf{0} & \mathbf{0} \\
\mathbf{0} & \boldsymbol{\Phi}_{\Delta \mu} & \mathbf{0} & \mathbf{0} & \mathbf{0} \\
\mathbf{0} & \mathbf{0} & \boldsymbol{\Phi}_{\alpha} & \mathbf{0} & \mathbf{0} \\
\mathbf{0} & \mathbf{0} & \mathbf{0} & \boldsymbol{\Phi}_{\phi} & \mathbf{1}_{4} \\
\mathbf{0} & \mathbf{0} & \mathbf{0} & \mathbf{0} & \boldsymbol{\Phi}_{\Delta \bar{\phi}}
\end{array}\right]
$$

where the submatrices $\boldsymbol{\Phi}_{i}$ are identity matrices of corresponding dimensions.

The discrete-time state transition equation can be expressed as

$$
\boldsymbol{\theta}_{k}=\boldsymbol{\Phi}_{k} \boldsymbol{\theta}_{k-1}+\mathbf{v}_{k}
$$

where $\mathbf{v}_{k} \sim \mathcal{N}(\mathbf{0}, \mathbf{Q})$ is the state noise with covariance matrix $\mathrm{Q}$. The discrete time state noise covariance matrix (for $P=1$ ) is defined as

$$
\mathbf{Q}_{\mid P=1}=\left[\begin{array}{ccc}
\mathbf{Q}_{\mu} & \mathbf{0} & \mathbf{0} \\
\mathbf{0} & \mathbf{Q}_{\alpha} & \mathbf{0} \\
\mathbf{0} & \mathbf{0} & \mathbf{Q}_{\phi}
\end{array}\right]
$$

where the submatrices are given by (8), (9), and (39) as

$$
\begin{aligned}
\mathbf{Q}_{\mu} & =\operatorname{diag}\left(\mathbf{q}_{\Delta \bar{\mu}}\right) \otimes\left[\begin{array}{cc}
\frac{1}{3} & \frac{1}{2} \\
\frac{1}{2} & 1
\end{array}\right] \\
\mathbf{Q}_{\alpha} & =q_{\alpha} \mathbf{I}_{4} \\
\mathbf{Q}_{\phi} & =\left[\begin{array}{ccccc}
q_{\phi_{\mathrm{HH}}} & q_{\phi_{C}} & q_{\phi_{C}} & q_{\phi_{C}} & q_{\phi_{\Delta C}} \\
q_{\phi_{C}} & q_{\phi_{\mathrm{HV}}} & q_{\phi_{C}} & q_{\phi_{C}} & q_{\phi_{\Delta C}} \\
q_{\phi_{C}} & q_{\phi_{C}} & q_{\phi_{\mathrm{VH}}} & q_{\phi_{C}} & q_{\phi_{\Delta C}} \\
q_{\phi_{C}} & q_{\phi_{C}} & q_{\phi_{C}} & q_{\phi_{\mathrm{V}}} & q_{\phi_{\Delta C}} \\
q_{\phi_{\Delta C}} & q_{\phi_{\Delta C}} & q_{\phi_{\Delta C}} & q_{\phi_{\Delta C}} & q_{\Delta \phi}
\end{array}\right]
\end{aligned}
$$

with $q_{\phi_{i}}=\left((1 / 3) q_{\Delta \phi}+q_{\phi}\right)$ for $i \in\{\mathrm{HH}, \mathrm{HV}, \mathrm{vH}, \mathrm{vV}\}, q_{\phi_{C}}=$ $(1 / 3) q_{\Delta \phi}$, and $q_{\phi_{\Delta C}}=(1 / 2) q_{\Delta \phi}$. The coefficients $1 / 3,1 / 2$, and 1 in (12)-(14) result from solving (38). The extension of the state equations (10) and (11) to multiple paths $(P>1)$ is obtained with the Kronecker product as $\boldsymbol{\Phi}=\boldsymbol{\Phi}_{\left.\right|_{P=1}} \otimes \mathbf{I}_{P}$, and $\mathbf{Q}=\mathbf{Q}_{\mid P=1} \otimes \mathbf{I}_{P}$

\section{B. Measurement Equations}

The structural parameters (2) are related to the channel sounder output through a complex shift operation [3]

$$
\mathbf{A}\left(\boldsymbol{\mu}_{i}\right)=\left[\begin{array}{ccc}
\mathrm{e}^{-\mathrm{j}\left(-\frac{N_{i}-1}{2}\right) \mu_{i, 1}} & \cdots & \mathrm{e}^{-\mathrm{j}\left(-\frac{N_{i}-1}{2}\right) \mu_{i, P}} \\
\vdots & & \vdots \\
\mathrm{e}^{-\mathrm{j}\left(+\frac{N_{i}-1}{2}\right) \mu_{i, 1}} & \cdots & \mathrm{e}^{-\mathrm{j}\left(+\frac{N_{i}-1}{2}\right) \mu_{i, P}}
\end{array}\right] \in \mathbb{C}^{N_{i} \times P} .
$$

The shift matrices $\mathbf{A}_{i}$ are multiplied by the corresponding system responses $\mathbf{G}_{i} \in \mathbb{C}^{M_{i} \times N_{i}}$ (provided by calibration measurements), yielding

$$
\begin{aligned}
\mathbf{B}_{f} & =\mathbf{G}_{f} \cdot \mathbf{A}\left(\boldsymbol{\mu}_{\tau}\right) \in \mathbb{C}^{M_{f} \times P} \\
\mathbf{B}_{R_{H}} & =\mathbf{G}_{R_{H}} \cdot\left(\mathbf{A}\left(\boldsymbol{\vartheta}_{\mathrm{R}}\right) \diamond \mathbf{A}\left(\boldsymbol{\varphi}_{\mathrm{R}}\right)\right) \in \mathbb{C}^{M_{\mathrm{R}} \times P} \\
\mathbf{B}_{R_{V}} & =\mathbf{G}_{R_{V}} \cdot\left(\mathbf{A}\left(\boldsymbol{\vartheta}_{\mathrm{R}}\right) \diamond \mathbf{A}\left(\varphi_{\mathrm{R}}\right)\right) \in \mathbb{C}^{M_{\mathrm{R}} \times P} \\
\mathbf{B}_{T_{H}} & =\mathbf{G}_{T_{H}} \cdot\left(\mathbf{A}\left(\boldsymbol{\vartheta}_{\mathrm{T}}\right) \oslash \mathbf{A}\left(\boldsymbol{\varphi}_{\mathrm{T}}\right)\right) \in \mathbb{C}^{M_{\mathrm{T}} \times P} \\
\mathbf{B}_{T_{V}} & =\mathbf{G}_{T_{V}} \cdot\left(\mathbf{A}\left(\boldsymbol{\vartheta}_{\mathrm{T}}\right) \oslash \mathbf{A}\left(\boldsymbol{\varphi}_{\mathrm{T}}\right)\right) \in \mathbb{C}^{M_{\mathrm{T}} \times P}
\end{aligned}
$$

where $\boldsymbol{\mu}_{\tau}=\left(2 \pi f_{s} / M_{f}\right) \cdot \boldsymbol{\tau}$ is the normalized delay ( $f_{0}$ being the sampling frequency). The system functions $\mathbf{G}_{(R / T)_{(H / V)}}$ for the antenna array responses are calculated from antenna calibration measurements using the effective aperture distribution function (EADF) [3], [9], [11]. The EADF is essentially a numerically efficient, algebraic differentiable representation of the antenna array manifold through a 2D-Fourier series expansion. To obtain the frequency response $\mathbf{G}_{f}$ of the system, e.g., a back-to-back cable calibration measurement is required.

As discussed in Section III-B, depending on the measurement setup and scenario, the (15) could also include a phasor due to the Doppler shift (see [3]). In our measurement model (15), this is not compensated for, due to assumed low mobility in the measured radio environment with respect to the relatively short time taken to measure all the antenna array ports (see Fig. 3).

Given the expressions for the basis functions (15), the matrix valued function $\mathbf{B}(\boldsymbol{\mu}) \in \mathbb{C}^{M \times 4 P}$ is defined as

$$
\begin{array}{r}
\mathbf{B}(\boldsymbol{\mu})=\left[\begin{array}{ll}
\mathbf{B}_{R_{H}} \diamond \mathbf{B}_{T_{H}} \diamond \mathbf{B}_{f} \quad \mathbf{B}_{R_{V}} \diamond \mathbf{B}_{T_{H}} \diamond \mathbf{B}_{f} \ldots \\
\mathbf{B}_{R_{H}} \diamond \mathbf{B}_{T_{V}} \diamond \mathbf{B}_{f} \quad \mathbf{B}_{R_{V}} \diamond \mathbf{B}_{T_{V}} \diamond \mathbf{B}_{f}
\end{array}\right] .
\end{array}
$$

Using (3) and (15), the propagation path parameters $\boldsymbol{\mu}$ and $\boldsymbol{\gamma}$ are mapped to an observation vector of length $M=M_{f} M_{\mathrm{T}} M_{\mathrm{R}}$ with the double-directional channel model [sampled version of (1)] as

$$
\mathbf{s}(\boldsymbol{\mu}, \gamma)=\mathbf{B}(\boldsymbol{\mu}) \cdot \boldsymbol{\gamma} \in \mathbb{C}^{M \times 1}
$$

The nonlinear measurement equation of the state-space model is given by

$$
\mathbf{y}_{k}=\mathbf{s}\left(\boldsymbol{\theta}_{k}\right)+\mathbf{r}_{y, k} \in \mathbb{C}^{M \times 1}
$$

where $\mathbf{s}\left(\boldsymbol{\theta}_{k}\right)$ is the mapping (17) of the propagation paths parameters to the observation, and $\mathbf{r}_{y, k} \sim \mathcal{C N}\left(\mathbf{0}, \mathbf{R}_{y, k}\right)$ is a colored noise process with a covariance matrix $\mathbf{R}_{y, k}$. 


\section{Measurement Noise Process: Receiver Noise and DMC}

The measurement noise covariance in the state-space model consists not only of i.i.d. second order ergodic Normal distributed measurement noise, but also of the dense multipath component (DMC) [3]. The DMC is caused by the various sources of distributed diffuse scattering in the radio channel. In earlier work [6], [7], [24]-[26] the DMC was assumed to be white in the angular domain, and to have structure (exponential decay) only in the delay domain, yielding

$$
\mathbf{R}_{a w}=\mathbf{I}_{M_{\mathrm{R}}} \otimes \mathbf{I}_{M_{\mathrm{T}}} \otimes \mathbf{R}_{f}+\sigma^{2} \mathbf{I}_{M}=\mathbf{I}_{M_{\mathrm{R}}} \otimes \mathbf{I}_{M_{\mathrm{T}}} \otimes \mathbf{R}_{f}^{\prime} .
$$

This model yields a computationally attractable solution to the EKF equations. This assumption holds for the distribution of scattered radio signals in e.g., many indoor scenarios. However, it is not generally valid in outdoor environments, e.g., street canyons, see [27]. The price to pay for this modelling inaccuracy is observed in the quality of the propagation path parameter estimates, which utilize the estimated DMC as the underlying noise process. Especially detection of new paths as well as path quality assessment suffer from the DMC model inaccuracy.

In this paper a further step is taken in the modelling of the DMC by allowing an unconstrained structure of the covariance matrices $\left(\mathbf{R}_{\mathrm{R}}\right.$ and $\left.\mathbf{R}_{\mathrm{T}}\right)$ at both ends of the measured radio channel. The only major assumptions regarding the structure of the measurement covariance matrix are, that the covariance of the DMC can be expressed as a Kronecker product of three matrices, namely the covariance matrices for the $\mathrm{Rx}\left(\mathbf{R}_{\mathrm{R}}\right)$, Tx $\left(\mathbf{R}_{\mathrm{T}}\right)$ and frequency $\left(\mathbf{R}_{f}\right)$ domains, and that there is additive i.i.d. Normal-distributed measurement noise present. This approach yields the expression for the shifted Kronecker structured measurement covariance matrix as

$$
\mathbf{R}=\mathbf{R}_{\mathrm{R}} \otimes \mathbf{R}_{\mathrm{T}} \otimes \mathbf{R}_{f}+\sigma^{2} \mathbf{I}_{M} .
$$

Fig. 6(a) shows the power-delay profile (PDP) of the residual $\mathbf{y}_{k}-\mathbf{s}\left(\hat{\boldsymbol{\theta}}_{k}\right)$ (after removing specular like path estimates) averaged over all $\left(M_{\mathrm{T}} \cdot M_{\mathrm{R}}\right)$ Tx-Rx channels. Two simulated PDPs are provided as well. The first simulation (approach 1) is using angular-white DMC. The proposed model (approach 2) [28] uses, as an example for the angular distribution, the von Mises-Fisher [29] distribution. Both of them fit well to the measured PDP in the delay domain [Fig. 6(a)], but Fig. 6(b) reveals significant difference in the angular domain. The approach 2 with von Mises-Fisher angular distribution is similar to the measured one, whereas the assumption on whiteness in angular domain (approach 1) is clearly not valid.

Solutions to the implementation challenges imposed by the shifted Kronecker structure are omitted here due to limited space (see [28] for details). The discussion in this paper concentrates on the estimation of the dominating propagation paths, and the elements of the measurement covariance are assumed to be obtained by another estimator [30].

\section{Propagation Path Parameter Estimation}

The proposed parameter estimation procedure is comprised of multiple estimators. The core of the algorithm, tracking the

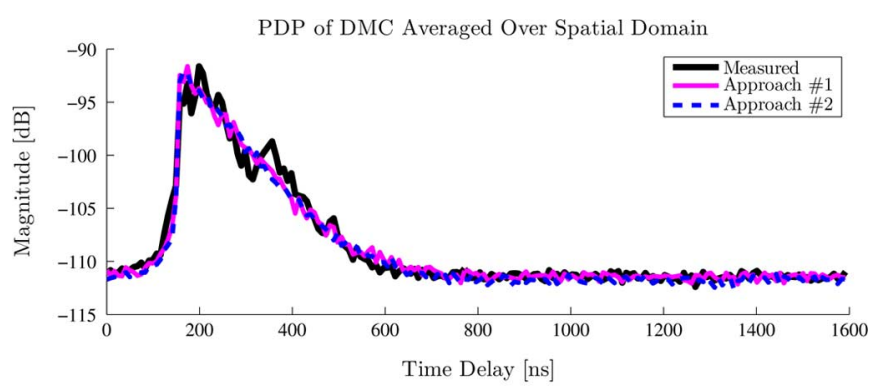

(a)

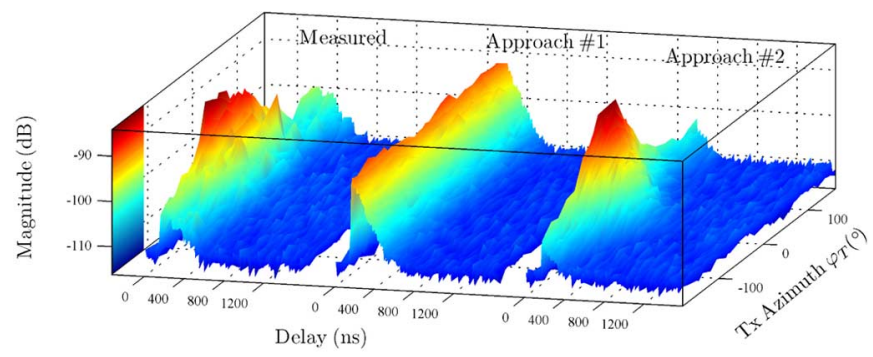

(b)

Fig. 6. Illustration of different approaches to model DMC. In the delay domain (a) both approaches 1 and 2 fit well to the measured DMC. In the angular domain (b) approach 1 clearly fails to model the measured DMC, whereas the proposed angular distribution (approach 2) fits well to the real-world measurement. (a) PDP of DMC in the delay domain (averaged over Rx and Tx channels). (b) Spatial PDP of DMC.

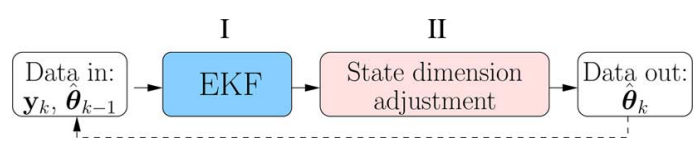

Fig. 7. Estimation procedure principle. The state vector $\hat{\boldsymbol{\theta}}_{k-1}$ (as well as other EKF system matrices) of previous time instant may have different dimensions than the current one $\hat{\boldsymbol{\theta}}_{k}$.

propagation path parameters over time, is based on the EKF. The approach for propagation path parameter estimation using Kalman filtering was introduced in [31]. One of the limitations of [31] is that the state dimension, which is proportional to the number of paths, was kept fixed. This is unrealistic, since new paths may appear and old paths disappear from observation to observation due to changes in the propagation environment or motion of the Tx or the Rx. Solving the resulting model order selection problem by a full log-likelihood based detection scheme [32] would be infeasible due to the large number of degrees of freedom involved. These issues were addressed in [6], [7], [24] along with other improvements to the state-space model. In this paper two one sided tests are proposed, one for detecting new paths (test for underfitting), and another for evaluating the significance of the tracked paths (test for overfitting). The tests for overfitting is applied after the EKF, and the path estimates for which the test statistics remain below the threshold are dropped from the state. The second test is applied for underfitting by searching new paths at each observation using a correlation-based grid detector. These tests are discussed in Section V-C. Also the DMC component, i.e., the covariance matrix $\mathbf{R}_{y, k}$, is estimated separately [30]. A block diagram of the developed estimation procedure is given in Fig. 7. 


\section{A. Extended Kalman Filter}

The propagation parameters are tracked using the Extended Kalman Filter. The EKF uses Taylor series expansion to linearize the nonlinear data model about the current estimates. To apply the EKF one needs to compute the first-order partial derivatives with respect to the parameters $\boldsymbol{\theta}$ of the data model $\mathbf{s}(\boldsymbol{\theta})$, i.e., the Jacobian matrix,

$$
\mathbf{D}(\boldsymbol{\theta})=\frac{\partial}{\partial \boldsymbol{\theta}^{\mathrm{T}}} \mathbf{s}(\boldsymbol{\theta})=\left[\frac{\partial}{\partial \theta_{1}} \mathbf{s}(\boldsymbol{\theta}) \cdots \frac{\partial}{\partial \theta_{L P}} \mathbf{s}(\boldsymbol{\theta})\right]
$$

which is derived for similar parameterization in [3]. To get compact expressions for the EKF, one can define the first order partial derivatives of the log-likelihood function of the measurement model $\mathcal{L}(\mathbf{y} \mid \boldsymbol{\theta}, \mathbf{R})$, namely the score function

$$
\begin{aligned}
\mathbf{q}(\mathbf{y} \mid \boldsymbol{\theta}, \mathbf{R}) & =\frac{\partial}{\partial \boldsymbol{\theta}} \mathcal{L}(\mathbf{y} \mid \boldsymbol{\theta}, \mathbf{R}) \\
& =2 \cdot \Re\left\{\mathbf{D}^{\mathrm{H}}(\boldsymbol{\theta}) \mathbf{R}^{-1}(\mathbf{y}-\mathbf{s}(\boldsymbol{\theta}))\right\}
\end{aligned}
$$

and the expected value of the second order partial derivative of $\mathcal{L}(\mathbf{y} \mid \boldsymbol{\theta}, \mathbf{R})$, the Fisher information matrix

$$
\begin{aligned}
\mathbf{J}(\boldsymbol{\theta}, \mathbf{R}) & =-\mathrm{E}\left\{\frac{\partial}{\partial \boldsymbol{\theta}} \mathcal{L}(\mathbf{y} \mid \boldsymbol{\theta}, \mathbf{R})\left(\frac{\partial}{\partial \boldsymbol{\theta}} \mathcal{L}(\mathbf{y} \mid \boldsymbol{\theta}, \mathbf{R})\right)^{\mathrm{T}}\right\} \\
& =2 \cdot \Re\left\{\mathbf{D}^{\mathrm{H}}(\boldsymbol{\theta}) \mathbf{R}^{-1} \mathbf{D}(\boldsymbol{\theta})\right\} .
\end{aligned}
$$

Using (21) and (22), the EKF equations can be formulated using the "Alternative form of the discrete Kalman filter" [33]. The results can be summarized as

$$
\begin{aligned}
\hat{\boldsymbol{\theta}}_{(k \mid k-1)} & =\boldsymbol{\Phi} \hat{\boldsymbol{\theta}}_{(k-1 \mid k-1)} \\
\mathbf{P}_{(k \mid k-1)} & =\boldsymbol{\Phi} \mathbf{P}_{(k-1 \mid k-1)} \boldsymbol{\Phi}^{\mathrm{T}}+\mathbf{Q} \\
\mathbf{P}_{(k \mid k)} & =\left(\mathbf{P}_{(k \mid k-1)}^{-1}+\mathbf{J}\left(\hat{\boldsymbol{\theta}}_{k \mid k-1}, \mathbf{R}_{y, k}\right)\right)^{-1} \\
\Delta \hat{\boldsymbol{\theta}}_{k} & =\mathbf{P}_{(k \mid k)} \mathbf{q}\left(\mathbf{y}_{k} \mid \hat{\boldsymbol{\theta}}_{(k \mid k-1)}\right) \\
\hat{\boldsymbol{\theta}}_{(k \mid k)} & =\hat{\boldsymbol{\theta}}_{(k \mid k-1)}+\Delta \hat{\boldsymbol{\theta}}_{k}
\end{aligned}
$$

where the Jacobian in (21) and (22) is evaluated at $\mathbf{D}_{k}=\mathbf{D}\left(\hat{\boldsymbol{\theta}}_{(k \mid k-1)}\right)$. One should note that this formulation of the EKF (23)-(27) does not explicitly solve for the Kalman gain. Instead, the filtering error covariance $\mathbf{P}_{(k \mid k)}$ (25) and filtered state estimate $\hat{\boldsymbol{\theta}}_{(k \mid k)}$ (27) are essentially updated in the information form. In addition to computational advantages, this formulation of the EKF with (21) and (22) allows the measurement $\mathbf{y}_{k}$, its covariance matrix $\mathbf{R}_{y, k}$ as well as the Jacobian matrix $\mathbf{D}_{k}$ to be complex valued.

Finding the direct numerical solution of (21) and (22) is i) computationally inefficient, and ii) often infeasible due to the large dimensions of $\mathbf{D}(\boldsymbol{\theta})$. Therefore, already in [3], algorithms have been developed to compute (21) and (22) without forming $\mathbf{D}(\boldsymbol{\theta})$ explicitly. They exploit the Tensor-structure of the data (16). However, the algorithms were derived using the simplified model for $\mathbf{R}$ (19) and are therefore not directly applicable for the problem at hand, which is using the generalized model for the noise-covariance matrix (20). Algorithms for computing $\mathbf{q}(\mathbf{y} \mid \boldsymbol{\theta})$ and $\mathbf{J}(\boldsymbol{\theta}, \mathbf{R})$ without forming explicitly the inverse of the shifted Kronecker-product $\left(\mathbf{R}^{-1}\right)$ of the DMC model (20), and the full matrix $\mathbf{D}(\boldsymbol{\theta})$ are described in [28].

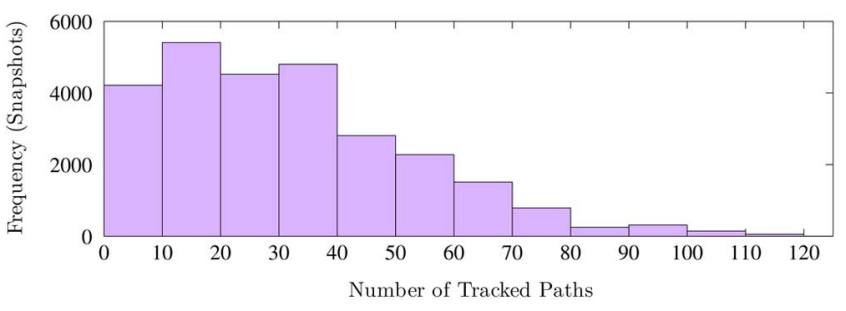

(a)

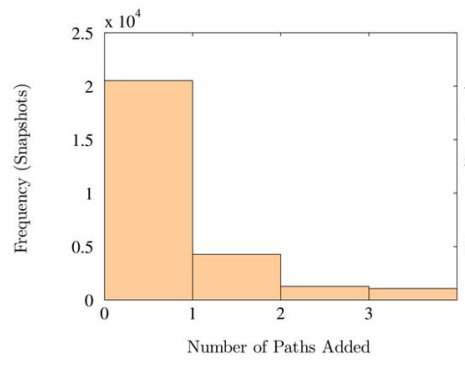

(b)

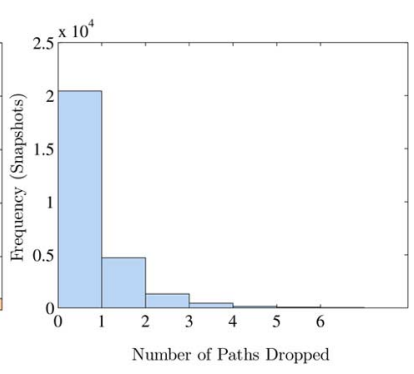

(c)
Fig. 8. Statistics for the number of paths tracked (a), added (b), and dropped (c) within a snapshot. The histograms stem from eight independent measurement routes and over 27000 snapshots.

\section{B. Reinitialization of Path Weights During Tracking}

If the number of polarization coefficients $N_{\text {pol }}$ is more than one, it may happen that the tracking of the phase of one or more polarization coefficients is lost since the individual SNR (relative to that of the dominating polarization) is low. This is due to the fact that the polarization coefficients may fade independently [see, e.g., Fig. 13(a)]. The weak polarization coefficients are denoted in the following by $\hat{\boldsymbol{\gamma}}_{w}$. The estimator (EKF) keeps track of the paths using the contribution of the polarization coefficients with high SNR, denoted in the following by $\hat{\gamma}_{s}$. Since all polarization coefficients of one path $p$ share the same $\bar{\phi}_{k, p}$, only the weak polarization coefficients $\hat{\gamma}_{w}$ must be reinitialized. ${ }^{1}$ For this purpose the best linear unbiased estimate (BLUE) for computing the polarization weights can be applied as [3]

$\hat{\gamma}_{w}=\left(\mathbf{B}^{\mathrm{H}}\left(\hat{\boldsymbol{\mu}}_{w}\right) \cdot \mathbf{R}_{y}^{-1} \cdot \mathbf{B}\left(\hat{\boldsymbol{\mu}}_{w}\right)\right)^{-1} \cdot \mathbf{B}^{\mathrm{H}}\left(\hat{\boldsymbol{\mu}}_{w}\right) \cdot \mathbf{R}_{y}^{-1} \cdot \tilde{\mathbf{y}}\left(\hat{\boldsymbol{\theta}}_{s}\right)$

where $\tilde{\mathbf{y}}\left(\hat{\boldsymbol{\theta}}_{s}\right)=\mathbf{y}-\mathbf{s}\left(\hat{\boldsymbol{\mu}}_{s}, \hat{\boldsymbol{\gamma}}_{s}\right)$, i.e., the effect of the dominating estimates has been removed from the observation. Also the values related to $\hat{\gamma}_{w}$ in the filtering error covariance matrix of the EKF should be reinitialized. The BLUE for the path weights (28) is also used for initializing new paths; see Section V-C.

\section{State Dimension Adjustment}

This section describes the procedure for choosing the state dimension, i.e., the number of paths $P$ to track. This is essentially a model order selection problem. In channel sounding the number of tracked paths may be over 100 [see Fig. 8(a)], and $P$ also changes slowly over time. An optimal solution to determine $P$ would be to compare all different path combinations using a likelihood-ratio test, but the number of combinations together

\footnotetext{
${ }^{1}$ The decision to reinitialize a coefficient can be based on e.g., thresholding the estimated phase variance provided by the filtering error covariance matrix.
} 
with the required computation time to evaluate a single likelihood function renders this approach infeasible.

In the following, a suboptimal solution using two one-sided statistical tests is proposed. This approach has proven to perform well in practice.

1) Initialization and Adding Paths to the State: Increasing the number of paths to track (the state dimension) is performed by a successive path cancellation (detect-estimate-cancel) principle, i.e., the contribution of the strongest estimated paths is removed until no more significant paths are detected.

The proposed detection scheme relies on a ML-based grid search. In [3] it was shown that the likelihood function of the measurement model (18) can be maximized by finding the structural parameters $\boldsymbol{\mu}$ that maximize

$$
\mathcal{C}\left(\boldsymbol{\mu}, \mathbf{y}, \mathbf{R}_{y}\right)=\mathbf{y}^{\mathrm{H}} \mathbf{R}_{y}^{-1} \mathbf{B}\left(\mathbf{B}^{\mathrm{H}} \mathbf{R}_{y}^{-1} \mathbf{B}\right)^{-1} \mathbf{B}^{\mathrm{H}} \mathbf{R}_{y}^{-1} \mathbf{y}
$$

where $\mathbf{B}=\mathbf{B}(\boldsymbol{\mu})$ is defined as (16), and $\mathbf{R}_{y}$ is defined as in (18) and (20). Equation (29) is expressed in terms of the structural parameters (2) only. For evaluating (29), a detection grid $\mu_{D}$ is formed. The grid consists of $D=D_{f} D_{\mathrm{T}} D_{\mathrm{R}}$ points in the space of the structural parameters (2). The detection grid should be selected such that it provides detection of a significant path from the entire parameter space (as a rule of thumb $D_{f} \approx 2 M_{f}, D_{\mathrm{T}} \approx 2 M_{\mathrm{T}}$, and $D_{\mathrm{R}} \approx 2 M_{\mathrm{R}}$ is sufficient). Then, using the most recent propagation path estimates $\hat{\boldsymbol{\theta}}_{k}$, the residual

$$
\tilde{\mathbf{y}}\left(\hat{\boldsymbol{\theta}}_{k}\right)=\mathbf{y}_{k}-\mathbf{s}\left(\hat{\boldsymbol{\mu}}_{k}, \hat{\boldsymbol{\gamma}}_{k}\right)
$$

is computed. To detect if there is a significant path present in the residual (30), the test statistic (29)

$$
C_{d}=2 \mathcal{C}\left(\boldsymbol{\mu}_{d}, \tilde{\mathbf{y}}\left(\hat{\boldsymbol{\theta}}_{k}\right), \mathbf{R}_{y, k}\right)
$$

is evaluated for each $d=1 \ldots D$ point in $\boldsymbol{\mu}_{D}$. The test hypotheses are $\mathbb{H}_{0}: \boldsymbol{\gamma}_{d}=0$ (path weight(s) corresponding to $\boldsymbol{\mu}_{d}$ is zero), and $\mathbb{H}_{1}: \boldsymbol{\gamma}_{d} \neq 0$ ( $\boldsymbol{\mu}_{d}$ has nonzero weight(s)). If the current model fits well to the data ( $\mathbb{H}_{0}$ holds), then $C_{d} \sim \chi_{2 N_{\mathrm{pol}}}^{2}$ (the values $C_{d}$ are $\chi^{2}$ distributed with $2 N_{\text {pol }}$ degrees of freedom). ${ }^{2}$ A new path is detected if the value $\max _{d}\left\{C_{d}\right\}$ exceeds a given threshold $\epsilon_{D}$, which can be determined for a specified probability of false alarm as

$$
p_{\mathrm{FA}, D}=\operatorname{Pr}\left(C_{d}>\epsilon_{D} \mid \mathbb{H}_{0}\right)=1-\int_{0}^{\epsilon_{D}} \chi_{2 N_{\mathrm{pol}}}^{2}(x) d x .
$$

The initial parameters $\hat{\boldsymbol{\theta}}_{k, n}$ for a new path are estimated by increasing the density of the grid in the vicinity of the detection point $\boldsymbol{\mu}_{d, \text { max }}$ and evaluating (29) for this denser grid. The structural parameters $\hat{\mu}_{k, n}$ corresponding to the maximum value in the denser grid are chosen as a new path estimate and the parameters $\hat{\boldsymbol{\theta}}_{k, n}=\left\{\hat{\boldsymbol{\mu}}_{k, n}, \hat{\boldsymbol{\gamma}}_{k, n}\right\}\left(\hat{\boldsymbol{\gamma}}_{k, n}\right.$ is given by (28) using $\left.\hat{\boldsymbol{\mu}}_{k, n}\right)$

${ }^{2} \mathbb{H}_{0}: C_{d} \sim \chi_{2 N_{\text {pol }}}^{2}$ can be shown by setting $\mathbf{B}^{\prime}=\mathbf{R}^{-(1 / 2)} \mathbf{B}$, and $\mathbf{y}^{\prime}=\mathbf{R}^{-(1 / 2)} \mathbf{y} \sim \mathcal{C} \mathcal{N}\left(\mathbf{0}, \mathbf{I} \mid \mathbb{H}_{0}\right)$ in (29), and by using properties of projection matrices [34]. are added to the state. Then the residual (30) is recomputed to cancel the contribution of the new path from the data. The $d e-$ tect-estimate-cancel steps are repeated until no additional significant path is detected. After the search, the variance values corresponding to the new detected paths $\hat{\boldsymbol{\theta}}_{k, N}$ in the EKF's filtering error covariance matrix (25) are initialized by evaluating the inverse of the observed Fisher information matrix (22) at $\mathbf{J}^{-1}\left(\hat{\boldsymbol{\theta}}_{k, N}, \mathbf{R}_{k}\right)$.

In practice, one should limit the number of new paths per snapshot to increase robustness against possible (temporary or systematic) model mismatches. Fig. 8 shows an example of the statistics on the number of paths $\hat{P}$ and the change of $\hat{P}$ in 8 measurement routes ( $>27000$ snapshots).

2) Reducing the Number of Paths: Removal of unreliable paths from the state is based on the Wald test [32]. The Wald test is not directly applicable for the parametrization of the path weights (4). Hence, the linear-scale path weight magnitudes are chosen as the test parameters $\boldsymbol{\theta}_{r}=\mathbf{a}_{p}=\left[\mathrm{e}^{\alpha_{\mathrm{HH}, p}} \cdots \mathrm{e}^{\alpha_{\mathrm{VV}, p}}\right]^{\mathrm{T}}$, with the test hypotheses for each path $p$ being $\mathbb{H}_{0}: \boldsymbol{\theta}_{r}=\mathbf{0}$ and $\mathbb{H}_{1}: \boldsymbol{\theta}_{r}>\mathbf{0}$. However, it can be shown (through the invariance property of Fisher information matrix under reparametrization [35]) that this is equivalent to path $p$ being valid (hypothesis $\mathbb{H}_{1}$ holding), when

$$
T_{W}=\hat{\boldsymbol{\theta}}_{r}^{\mathrm{T}}\left(\left[\mathbf{P}_{k}\right]_{\theta_{r} \theta_{r}}\right)^{-1} \hat{\boldsymbol{\theta}}_{r}=\mathbf{1}^{\mathrm{T}}\left(\left[\mathbf{P}_{k}\right]_{\alpha_{p} \alpha_{p}}\right)^{-1} \mathbf{1}>\epsilon_{S}
$$

where $\left[\mathbf{P}_{k}\right]_{\alpha_{p} \alpha_{p}}$ is the filtering error covariance matrix corresponding to the columns and rows related to parameters $\boldsymbol{\alpha}_{r}$, and $\left[\mathbf{P}_{k}\right]_{\theta_{r} \theta_{r}} \in \mathbb{R}^{N_{r} \times N_{r}}$ is the one transformed for the linear-scale parameters $\boldsymbol{\theta}_{r}$. If the path is insignificant $\left(\mathbb{H}_{0}\right.$ holds), then $T_{W} \sim$ $\chi_{N_{\mathrm{pol}}}^{2}$ is $\chi^{2}$ distributed with $N_{\mathrm{pol}}$ degrees of freedom. Thus, $\epsilon_{S}$ can be chosen through the probability of falsely deciding an insignificant path to be significant as

$$
p_{\mathrm{FA}, S}=\operatorname{Pr}\left(T_{W}>\epsilon_{S} \mid \mathbb{H}_{0}\right)=1-\int_{0}^{\epsilon_{S}} \chi_{N_{\mathrm{pol}}}^{2}(x) d x .
$$

For the data model with $N_{\mathrm{pol}}=4$, and $p_{\mathrm{FA}_{S}}=0.01$, the threshold would be $\epsilon_{S}=13.3$.

It should be noted that both tests have computational complexity low enough to run in a reasonable processing time alongside the EKF. A flow chart summarizing the implemented algorithm is presented in Fig. 9.

\section{ESTIMATION EXAMPLES}

The algorithm has been implemented and tested with measurement data from downtown Ilmenau, Germany. The measurements were conducted with the RUSK Channel Sounder [10] by Technical University of Ilmenau in 2004 [14]. The measurement parameters are given in Table I, and the map of the measurements is shown in Fig. 10.

A visualization of the algorithm performance is shown in Fig. 11. The individual plots in Fig. 11 illustrate the powerangular-delay profiles (PADPs) of the full measurement $\left(\mathbf{y}_{k}\right)$, as well as the PADP of the residual after removing the estimated propagation paths $\left(\tilde{\mathbf{y}}_{k}=\mathbf{y}_{k}-\mathbf{s}\left(\boldsymbol{\theta}_{k}\right)\right)$, and the PADP of the whitened residual $\left(\tilde{\mathbf{y}}_{w, k}=\mathbf{R}^{-(1 / 2)} \tilde{\mathbf{y}}_{k}\right)$ respectively. The 


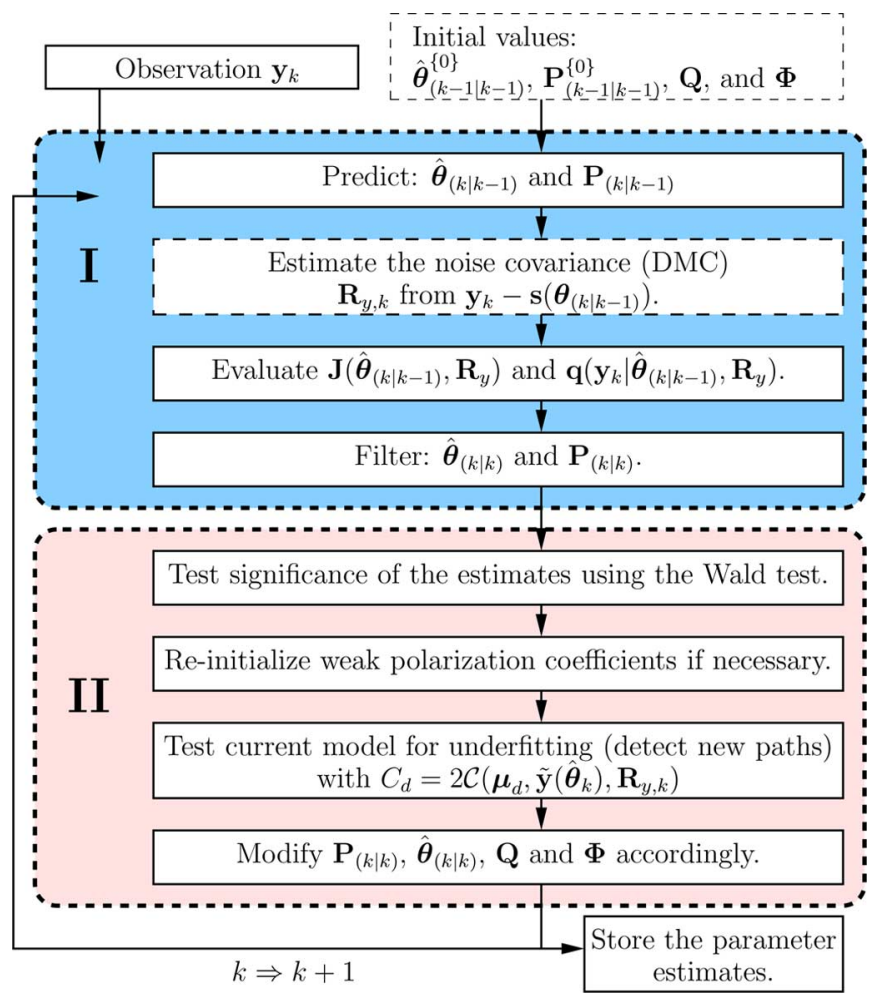

Fig. 9. Complete estimation procedure. The higher level concepts in Fig. 7 are marked with areas I and II.

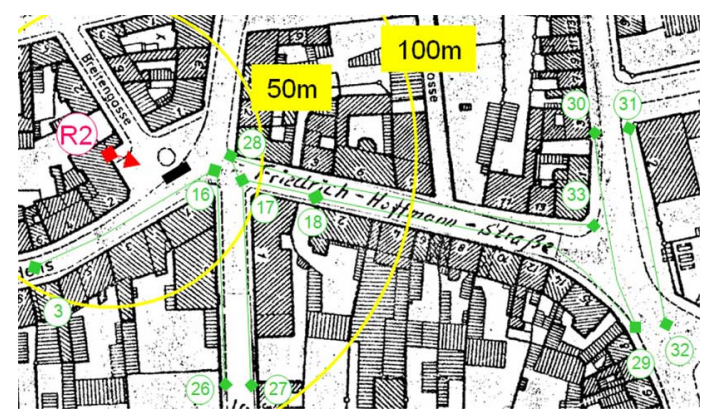

Fig. 10. Map of the measured Tx routes at downtown Ilmenau. $R 2$ denotes the position of the receiver.

TABLE I

MEASUREMENT PARAMETERS

\begin{tabular}{|r|l|}
\hline Center Freq. & $5.2 \mathrm{GHz}$ \\
\hline Bandwidth & $120 \mathrm{MHz}$ \\
\hline$M_{f} / \Delta t$ & 193 points/20.48 ms \\
\hline Rx array & 8 element ULA (dual-polarized, $16\left(=M_{R}\right)$ ports $)$ \\
\hline Tx array & $16\left(=M_{T}\right)$ element UCA \\
\hline
\end{tabular}

PADPs are computed using beamforming for visualizing the angular spectra yielding

$$
S_{\mathrm{T}}\left(\varphi_{\mathrm{T}}\right)=\frac{1}{M_{\mathrm{R}}} \sum_{m_{\mathrm{R}}=1}^{M_{\mathrm{R}}} \frac{\mathbf{b}\left(\varphi_{\mathrm{T}}, \vartheta_{T, 0}\right)^{\mathrm{H}}}{\sqrt{\mathbf{b}\left(\varphi_{\mathrm{T}}, \vartheta_{T, 0}\right)^{\mathrm{H}} \mathbf{b}\left(\varphi_{\mathrm{T}}, \vartheta_{T, 0}\right)}} \mathbf{Y}_{m_{\mathrm{R}}}
$$

where $\mathbf{Y}_{m_{\mathrm{R}}} \in \mathbb{C}^{M_{\mathrm{T}} \times M_{f}}$ denotes the impulse responses of all the Tx channels and the $m_{\mathrm{R}}{ }^{\text {th }} \mathrm{Rx}$ channel, and $\mathrm{b}\left(\varphi_{\mathrm{T}}, \vartheta_{T, 0}\right)$ is

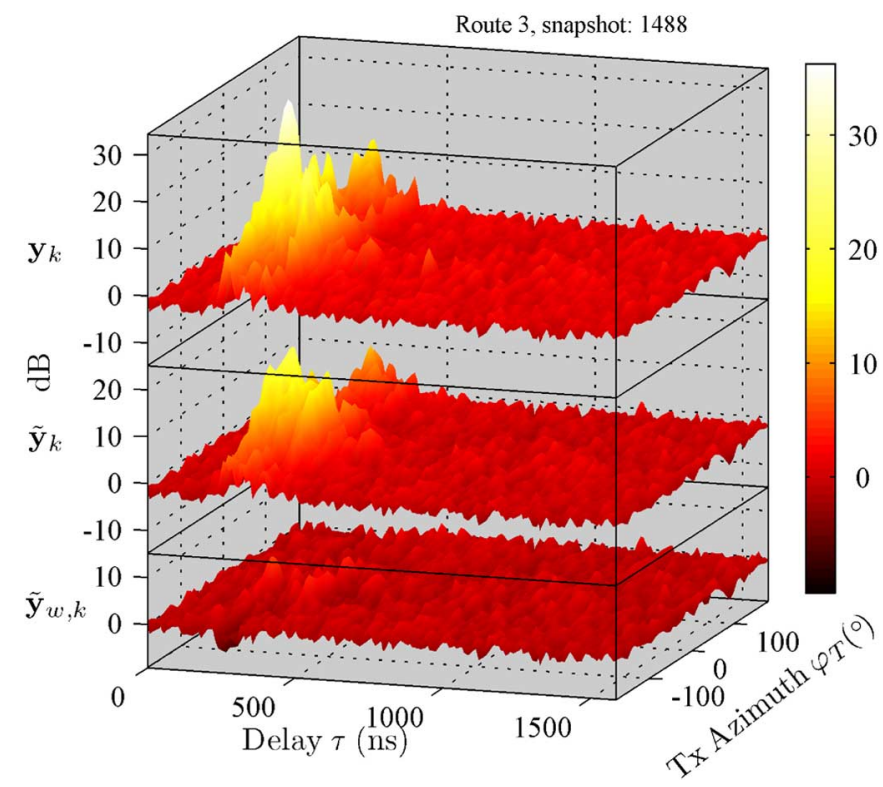

Fig. 11. Power-Tx azimuth angle-delay profile of a measurement $\left(\mathbf{y}_{k}\right)$, compared with the residual after removing estimated propagation paths $\left(\tilde{\mathbf{y}}_{k}=\right.$ $\left.\mathbf{y}_{k}-\mathbf{s}\left(\boldsymbol{\theta}_{k}\right)\right)$ and the whitened residual $\left(\tilde{\mathbf{y}}_{w, k}=\mathbf{R}^{-(1 / 2)} \tilde{\mathbf{y}}_{k}\right)$. The profiles are obtained via beamforming, and are averaged over all Rx channels.

the Tx steering vector (calibrated response of the antenna array) for the angles $\varphi_{\mathrm{T}}$ and $\vartheta_{T, 0}$. The Hann-window was applied in the discrete Fourier transformation to estimate the impulse responses from the frequency domain data. For illustration purposes, the PADPs of $\mathbf{y}_{k}$ and $\tilde{\mathbf{y}}_{k}$ are scaled such that the average noise power is $0 \mathrm{~dB}$. The lowest plot shows that the whitened residual indeed resembles white noise.

Fig. 12 shows the delay [Fig. 12(a)] and Tx azimuth angle [Fig. 12(b)] estimates of all paths, as well as the total number of estimated paths over time [Fig. 12(c)] from the route between the points 17 and 29 in Fig. 10. The sum power of both polarizations (in decibels) is indicated by the color scale.

Fig. 13 illustrates the estimates of the path weight components of a single path over about 450 snapshots. The figures show the magnitude [Fig. 13(a)], as well as the evolution of the phase [Fig. 13(b)], and the phase prediction error [Fig. 13(c)] for the vertical-to-vertical (VV) and vertical-to-horizontal (VH) polarization components. The solid line in Fig. 13(b) denotes the estimated $\Delta \bar{\phi}$ parameter. The phase evolution of both polarization components in Fig. 13(b) clearly has the same trend $(\Delta \bar{\phi})$, which supports the assumption of a common $\Delta \bar{\phi}$ for both components (related to the Doppler shift, see Section III-B). Fig. 13(c) shows the difference between predicted and filtered path weight phase estimates. The solid and dashed lines denote the estimated (by EKF) $\pm \sigma$ confidence intervals for both VV and $\mathrm{VH}$ polarizations. Comparing these $\sigma$ curves to the magnitudes in Fig. 13(a), it can be observed that the phase error estimate is clearly higher at points where the magnitude of the corresponding ( $\mathrm{VV}$ or $\mathrm{VH}$ ) component experiences deeper fading (e.g., after $21 \mathrm{~s}$ for $\mathrm{VH}$ polarization and at about $18 \mathrm{~s}$ for $\mathrm{VV}$ polarization).

Fig. 14 shows the tracks of estimated delay and Tx azimuth angle for a single path among the estimates in Fig. 12. Based on the estimates, this particular path is being tracked while the 


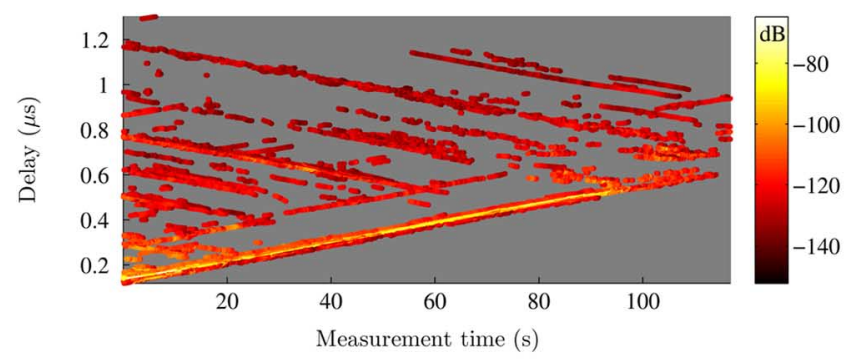

(a)

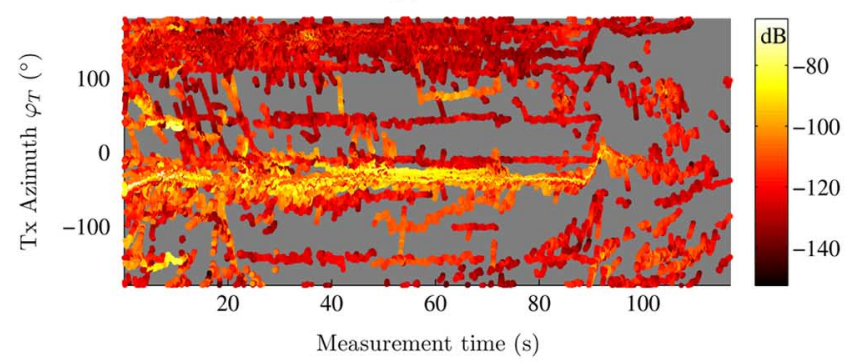

(b)

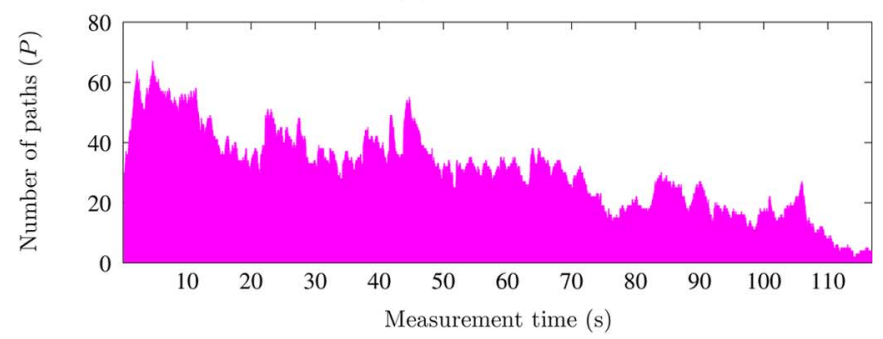

(c)

Fig. 12. Example plots of the discrete path delay (a) and Tx azimuth (b) estimates as well as the total number of tracked paths (c) from the route (of more than 5000 snapshots) between the points 17 and 29 in Fig. 10. The color indicates the sum power of polarization components. Both, the line of sight (bright yellow, increasing delay, $\varphi_{\mathrm{T}} \approx-20^{\circ}$ ) and reflected paths (red, decreasing delay, $\varphi_{\mathrm{T}} \neq-20^{\circ}$ ) can be identified. The line of sight path is lost at $t \approx 95 \mathrm{~s}$ as the Tx moves behind a corner.

Tx moves besides a point of reflection. Fig. 14(a) also indicates a clear relationship between the change in the propagation delay of a path and the evolution of the path weight phase [see also (5)].

\section{CONCLUSION}

This paper introduces a novel approach to the sequential estimation of propagation path parameters from MIMO channel sounding measurements. A state-space model including all path parameters is developed, and a computationally attractive form of the EKF is introduced, allowing scalability in terms of both the state and measurement dimensions. Furthermore, a statistical approach is introduced to detect new propagation paths as well as to remove disappeared paths. The importance of the path weight parametrization, as well as a proper phase prediction is addressed. In addition, introducing unconstrained Rx and Tx covariance matrices for the DMC process improves the estimation of the propagation path parameter estimates, and enhances the reliability of the model order selection (detection and removal of paths). The estimation examples support the applicability of the proposed methods for capturing the dynamic behavior of MIMO

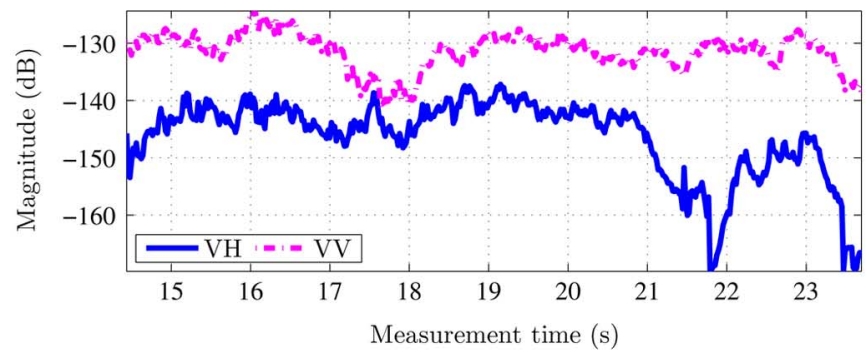

(a)

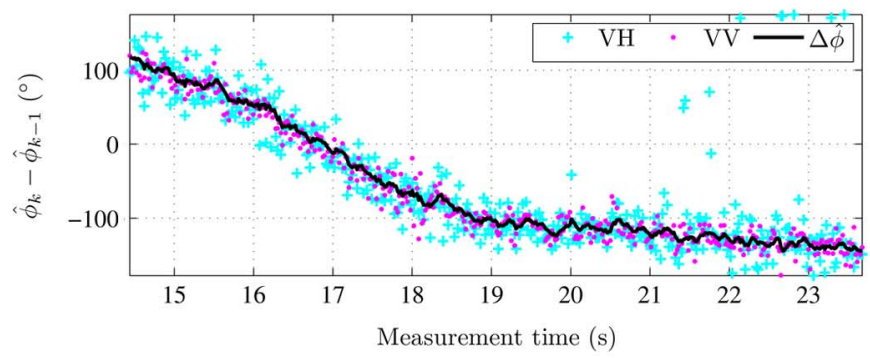

(b)

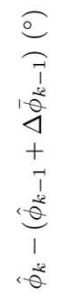

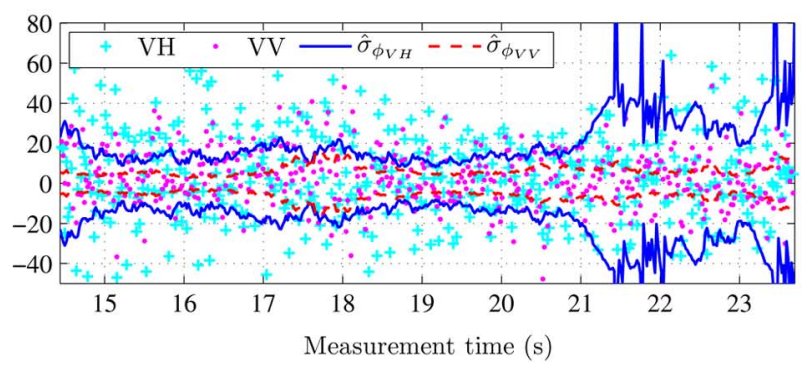

(c)

Fig. 13. Example of an estimated path weight from a real measurement. Figures show the magnitude (a), as well as the evolution of the phase (b), and the phase prediction error (c) for a single path tracked for over 800 snapshots.

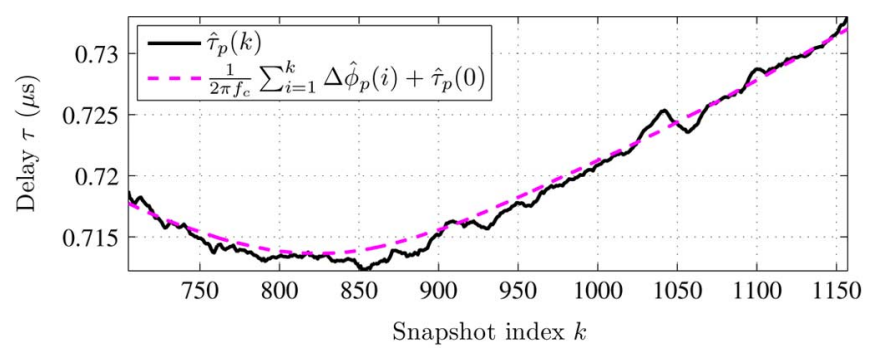

(a)

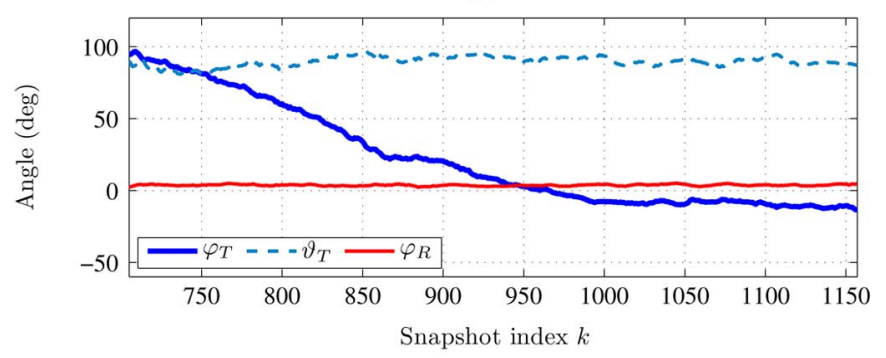

(b)

Fig. 14. Examples of the parameter estimates of a single path. The tracks of the delay $\tau$ (a) as well as the Tx azimuth angle $\varphi_{\mathrm{T}}$ (b) estimates indicate that the path was tracked while Tx was passing some point of reflection. The fluctuation in the track of the delay $\tau$ may be caused by the $q_{\Delta \bar{\tau}}$ value being too low (for this particular path), leading to slow tracking. In (a) also the relation (5) of the path weight phase change parameter $\Delta \bar{\phi}$ to the delay $\tau$ is illustrated. 
channels, which is crucial in developing realistic channel models for mobile MIMO communication systems.

\section{APPENDIX \\ MATRIX FRACTION DECOMPOSITION FOR DisCRETIZING THE STATE EQUATIONS}

The discrete state transition and state noise covariance matrices can be obtained as follows [21]. The continuous time differential equation (8) can be written as

$$
\frac{\mathrm{d} \boldsymbol{\theta}(t)}{\mathrm{d} t}=\mathbf{F} \boldsymbol{\theta}(t)+\mathbf{L w}(t) .
$$

Given the matrices $\mathbf{F}$ and $\mathbf{L}$ (see (8) and (36)) as well as the power spectral density of the noise $\mathbf{W}(9)$, the discrete time state transition matrix is defined as

$$
\mathbf{\Phi}=\exp (\mathbf{F} \Delta t)
$$

and the state noise covariance matrix as

$$
\mathbf{Q}=\int_{0}^{\Delta t} \exp (\mathbf{F}(\Delta t-\tau)) \mathbf{L} \mathbf{W} \mathbf{L}^{\mathrm{T}} \exp (\mathbf{F}(\Delta t-\tau))^{\mathrm{T}} \mathrm{d} \tau
$$

The matrix $\mathbf{Q}$ can be solved efficiently using the following matrix fraction decomposition [21]

$$
\begin{aligned}
{\left[\begin{array}{l}
\mathbf{C} \\
\mathbf{D}
\end{array}\right] } & =\exp \left(\left[\begin{array}{cc}
\mathbf{F} & \mathbf{L W L} \\
\mathbf{0} & -\mathbf{F}^{\mathrm{T}}
\end{array}\right] \Delta t\right)\left[\begin{array}{l}
\mathbf{0} \\
\mathbf{I}
\end{array}\right] \\
\mathbf{Q} & =\mathbf{C D}^{-1} .
\end{aligned}
$$

\section{REFERENCES}

[1] M. Steinbauer, A. Molisch, and E. Bonek, "The double-directional radio channel," IEEE Antennas Propag. Mag., vol. 43, no. 4, pp. 51-63, Aug. 2001.

[2] B. H. Fleury, M. Tschudin, R. Heddergott, D. Dahlhaus, and K. I. Pedersen, "Channel parameter estimation in mobile radio environments using the SAGE algorithm," IEEE J. Sel. Areas Commun., vol. 17, no. 3, pp. 434-450, Mar. 1999.

[3] A. Richter, "Estimation of radio channel parameters: Models and algorithms" Ph.D. dissertation, Technischen Universität Ilmenau, Ilmenua, Germany, May 2005 [Online]. Available: www.db-thueringen.de

[4] N. Czink, R. Tian, S. Wyne, F. Tufvesson, J.-P. Nuutinen, J. Ylitalo, E. Bonek, and A. Molisch, "Tracking time-variant cluster parameters in MIMO channel measurements," in Proc. 2nd Int. Conf. Communications Networking in China (CHINACOM), Aug. 2007, pp. 1147-1151.

[5] X. Yin, G. Steinbock, G. Kirkelund, T. Pedersen, P. Blattnig, A. Jaquier, and B. Fleury, "Tracking of time-variant radio propagation paths using particle filtering," in Proc. IEEE Int. Conf. Communications (ICC'08), May 2008, pp. 920-924.

[6] J. Salmi, A. Richter, M. Enescu, P. Vainikainen, and V. Koivunen, "Propagation parameter tracking using variable state dimension Kalman filter," presented at the IEEE Vehicular Technology Conf. (VTC) 2006 Spring, Melbourne, Australia, May 7-10, 2006.

[7] J. Salmi, A. Richter, and V. Koivunen, "Enhanced tracking of radio propagation path parameters using state-space modeling," presented at the EUSIPCO, Florence, Italy, Sep. 2006.

[8] D. Tse and P. Viswanath, Fundamentals of Wireless Communications. Cambridge, U.K.: Cambridge Univ. Press, 2005.

[9] Smart Antennas-State of the Art, T. Kaiser, A. Bourdoux, H. Boche, J. R. Fonollosa, J. B. Andersen, and W. Utschick, Eds. New York: Hindawi Publishing Corp., 2005, ch. 13.
[10] Channelsounder.de, MEDAV GmbH, [Online]. Available: http://www. channelsounder.de/

[11] M. Landmann, A. Richter, and R. Thomä, "DOA resolution limits in MIMO channel sounding," in Proc. Int. Symp. Antennas Propagation and USNC/URSI Nat. Radio Science Meeting, Monterey, CA, Jun. 2004, pp. 1708-1711.

[12] Mobile Broadband Multimedia Networks, L. M. Correia, Ed., 1st ed. Norwell, MA: Academic, May 2006.

[13] P. J. Huber, Robust Statistics. New York: Wiley, 1981.

[14] U. Trautwein, M. Landmann, G. Sommerkorn, and R. Thomä, "System-oriented measurement and analysis of MIMO channels," in COST273 12th Management Committee Meeting, Bologna, Italy, Jan. 19-21, 2005, TD $(05) 063$.

[15] P. Almers, S. Wyne, F. Tufvesson, and A. Molisch, "Effect of random walk phase noise on MIMO measurements," in Proc. IEEE Vehicular Technology Conf. (VTC) 2005 Spring, Stockholm, Sweden, May 2005, vol. 1 , pp. 141-145.

[16] A. Taparugssanagorn, "Evaluation of MIMO radio channel characteristics from TDM-Switched MIMO channel sounding" Ph.D. Dissertation, Univ. of Oulu, Oulu, Finland, 2007 [Online]. Available: http:// herkules.oulu.fi/isbn9789514286506/isbn9789514286506.pdf

[17] X. Yin, "High-resolution parameter estimation for MIMO channel sounding," Ph.D. dissertation, Aalborg Univ., Aalborg, Denmark, 2006.

[18] R. H. Shumway and D. S. Stoffer, Time Series Analysis and Its Applications With R Examples. New York: Springer, 2006.

[19] T. K. Y. Bar-Shalom and X. R. Li, Estimation with Applications to Tracking and Navigation. New York: Wiley, 2001, ch. 6.

[20] J. M. Mendel, Lessons in Estimation Theory for Signal Processing Communications, and Control. Englewood Cliffs, NJ: Prentice-Hall, 1995.

[21] J. Hartikainen and S. Särkkä, EKF/UKF Toolbox for Matlab Espoo, Finland, Tech. Rep., 2008 [Online]. Available: http://www.lce.hut.fi/ research/mm/ekfukf/

[22] S. Särkkä, "Recursive Bayesian inference on stochastic differential equations" Ph.D. dissertation, Helsinki Univ. of Technology, Espoo, Finland, 2006 [Online]. Available: http://lib.tkk.fi/Diss/

[23] M. S. Grewal and A. P. Andrews, Kalman Filtering, Theory and Practice Using MATLAB. New York: Wiley-Interscience, 2001.

[24] J. Salmi, A. Richter, and V. Koivunen, "State-space modeling and propagation parameter tracking: Multitarget tracking based approach," in Proc. 40th Asilomar Conf. Signals, Systems, Computers, Pacific Grove, CA, Oct. 2006, pp. 941-945.

[25] A. Richter, J. Salmi, and V. Koivunen, "On distributed scattering in radio channels and its contribution to MIMO channel capacity," presented at the 1st Eur. Conf. Antennas Propagation, Nice, France, Nov. 2006.

[26] A. Richter, J. Salmi, and V. Koivunen, "An algorithm for estimation and tracking of distributed diffuse scattering in mobile radio channels," presented at the IEEE Int. Workshop Signal Processing Advances Wireless Communications, Cannes, France, Jul. 2-5, 2006.

[27] A. Richter, J. Salmi, and V. Koivunen, "Signal processing perspectives to radio channel modelling," presented at the 2 nd Eur. Conf. Antennas Propagation (EuCAP 2007), Edinburgh, U.K., Nov. 11-16, 2007.

[28] J. Salmi, A. Richter, and V. Koivunen, "Tracking of MIMO propagation parameters under spatio-temporal scattering model," in Proc. 41 st Asilomar Conf. Signals, Systems, Computers, Pacific Grove, CA, Nov. 2007, pp. 666-670

[29] K. V. Mardia, Statistics of Directional Data. London, U.K.: Academic, 1972

[30] A. Richter, J. Salmi, and V. Koivunen, "ML estimation of covariance matrix for tensor valued signals in noise," presented at the IEEE Int. Conf. Acoustics, Speech, Signal Processing, Las Vegas, NV, Apr. 2008.

[31] A. Richter, M. Enescu, and V. Koivunen, "State-space approach to propagation path parameter estimation and tracking," presented at the 6th IEEE Workshop on Signal Processing Advances in Wireless Communications, New York, Jun. 2005

[32] S. M. Kay, Fundamentals of Statistical Signal Processing: Detection Theory. Englewood Cliffs, NJ: Prentice-Hall Int., 1998, vol. 2.

[33] R. G. Brown and P. Y. Hwang, Introduction to Random Signals and Applied Kalman Filtering, 3rd ed. New York: Wiley, 1997.

[34] G. H. Golub and C. F. van Loan, Matrix Computations, 3rd ed. Baltimore, MD: The Johns Hopkins Univ. Press, 1996.

[35] L. Scharf, Statistical Signal Processing, Detection Estimation and Time Series Analysis. Reading, MA: Addison-Wesley, 1990. 


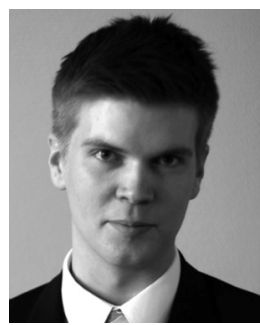

Jussi Salmi (S'05) was born in Finland in 1981. $\mathrm{He}$ received the M.Sc. degree (with hons.) from the Department of Electrical and Communications Engineering, Helsinki University of Technology, Espoo, Finland, in 2005, where he is currently working towards the Ph.D. degree.

From 2004 to 2005, he worked as a Research Assistant at Radio Laboratory, Helsinki University of Technology. Since 2005, he has been a Researcher at the Department of Signal Processing and Acoustics, Helsinki University of Technology. Since 2007 he has been a member of Graduate School in Electronics, Telecommunications and Automation (GETA). His current research interests include measurement based MIMO channel modeling, parameter estimation, analysis of interference limited multiuser MIMO measurements as well as tensor modeling and decomposition techniques.

Mr. Salmi received the Best Student Paper Award in EUSIPCO 2006 for a paper he authored and a Best Paper Award in Propagation for a paper he coauthored in EuCAP 2006.

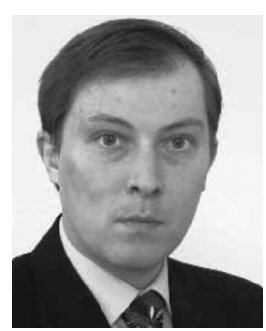

Andreas Richter (M'04-SM'08) was born in Germany in 1969. He received the Dipl.-Ing. (M.Sc.) degree in electrical engineering and the Dr.-Ing. (Ph.D.) degree (summa cum laude) from Technische Universität Ilmenau, Ilmenau, Germany, in 1995 and 2005, respectively.

From 1995 to 2004, he worked as a Research Assistant at the Electronic Measurement Laboratory of Technische Universität Ilmenau. From July to October 2001, he was a Guest Researcher at NTT DoCoMo's, Wireless Laboratories, Yokosuka, Japan. From 2004 to 2008, he was a Senior Research Fellow in the Statistical Signal Processing Laboratory at Helsinki University of Technology, Finland. Since August 2008, he has been a Principal Member of Research Staff at Nokia Research Center, Helsinki. His research interests are in the fields of digital communication, sensor array, and statistical signal processing. He has published more than 70 peer-reviewed papers in international scientific conferences and journals.

Dr. Richter was coauthor or author of five papers receiving a Best Paper Award (EPMCC 2001, ISAP 2004, PIMRC 2005, EUSIPCO 2006, and EuCAP 2006). In 2005, he received the Siemens Communications Academic Award. $\mathrm{He}$ and his former colleagues at Technische Universität Ilmenau received the Thuringian Research Award for Applied Research in 2007 for their work on MIMO channel sounding.

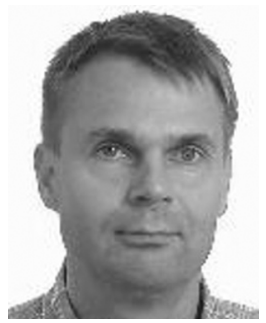

Visa Koivunen (S'87-M'93-SM'98) received the D.Sc. (Tech.) degree (with hons.) from the Department of Electrical Engineering, University of Oulu, Finland.

From 1992 to 1995, he was a Visiting Researcher at the University of Pennsylvania, Philadelphia. In 1996, he held a faculty position at the Department of Electrical Engineering, University of Oulu. From August 1997 to August 1999, he was an Associate Professor at the Signal Processing Laboratory, Tampere University of Technology, Finland. Since 1999, he has been a Professor of signal processing at the Department of Electrical and Communications Engineering, Helsinki University of Technology (HUT), Finland. He is one of the Principal Investigators in SMARAD (Smart Radios and Wireless Systems) Center of Excellence in Radio and Communications Engineering nominated by the Academy of Finland. Since 2003, he has been also Adjunct Full Professor at the University of Pennsylvania, Philadelphia. During his sabbatical leave in 2006-2007, he was Visiting Fellow at Nokia Research Center as well as a Visiting Professor at Princeton University, Princeton, NJ. His research interests include statistical, communications, and sensor array signal processing. He has published more than 260 papers in international scientific conferences and journals.

Dr. Koivunen received the Primus Doctor (best graduate) Award among the doctoral graduates during the years 1989 to 1994 . He coauthored the papers receiving the Best Paper Award in IEEE PIMRC 2005, EUSIPCO 2006, and EuCAP 2006. He has been awarded the IEEE Signal Processing Society Best Paper Award for 2007 (for a paper coauthored with J. Eriksson). He served as an Associate Editor for the IEEE SIGNAL PROCESSING LETTERS. He is a member of the Editorial Board for the Signal Processing journal and the Journal of Wireless Communication and Networking. He is also a member of the IEEE Signal Processing for Communication Technical Committee (SPCOM-TC). He was the General Chair of the IEEE Signal Processing Advances in Wireless Communication (SPAWC) Conference in Helsinki, Finland, in June 2007. 